\title{
Review Article \\ Fluorescent Gold Nanoclusters: Synthesis and Recent Biological Application
}

\author{
Xiaochao Qu, Yichen Li, Lei Li, Yanran Wang, Jingning Liang, and Jimin Liang \\ School of Life Science and Technology, Xidian University, Xi'an, Shannxi 710071, China \\ Correspondence should be addressed to Xiaochao Qu; xiaochaoqu@gmail.com \\ Received 16 September 2014; Accepted 14 January 2015 \\ Academic Editor: Rajesh Naik \\ Copyright ( 2015 Xiaochao Qu et al. This is an open access article distributed under the Creative Commons Attribution License, \\ which permits unrestricted use, distribution, and reproduction in any medium, provided the original work is properly cited.

\begin{abstract}
Fluorescent gold nanoclusters (AuNCs) have been extensively studied due to their unique construction and distinctive properties, which place them between single metal atoms and larger nanoparticles. The dimension of AuNCs is comparable to the Fermi wavelength of electrons, which lead to size-dependent fluorescence and other molecule-like properties. In this review, we summarize various synthesis strategies of fluorescent AuNCs and recent advances of biological applications such as biosensing, biolabeling, and bioimaging. The synthetic methods are considered as two routes: "Atoms to Clusters" and "Nanoparticles to Clusters." The surface functionalization of AuNCs is described as the precondition for making future bioapplications possible, which can eventually influence their stability, biocompatibility, and other properties. And then we focus on the recent advances of AuNCs-based applications in biological sensing, biolabeling, and bioimaging and finally discuss the current challenges of AuNCs in controllable synthesis and biological application.
\end{abstract}

\section{Introduction}

The word "nano" is for describing something which occurs with size on nanometer range [1]. The worldwide emergence of nanoscale technology and engineering was marked by the announcement of the National Nanotechnology Initiative (NNI) in January 2000 [2]. Nanotechnology, which became an emerging and promising research field in the past decades, is the combination of medicine, chemistry, physics, and biology [3]. This multidisciplinary technology is considered dedicating to assemble molecules into objects and reverse this process. The confluence of biology and nanotechnology is defined as nanobiotechnology, which is a field that investigates biosystems and creates new biomaterials using nanoscale principles and techniques [2]. The nanostructure materials in biology and biomedical field can be useful tools for biosensing, cell labeling, and molecular imaging.

Gold is the most extensively studied material among worldwide groups due to its stable chemical property, facile synthesis, and nontoxicity. Gold on bulk- or molecular-range is highly stable; however, gold nanoparticles (NPs) exhibit distinctive properties which differ from others such as their surface plasmon resonance (SPR) effect, size-depending electronic properties, and their photothermal effect in biological therapy [4]. For these fascinating aspects, gold NPs have been considered as the key materials of nanoscience and nanotechnology in recent years. Due to quantum confinement effects of gold nanoparticles with a dimension below $2 \mathrm{~nm}$, they are too small to support surface plasmon resonance effect [5]. The noble metal nanomaterials (which we call them gold nanoclusters) are composed of several to roughly a hundred atoms $[6,7]$. The dimension occurring with gold nanoclusters (AuNCs) is comparable to the Fermi wavelength of electrons [8], which place them between single metal atoms and larger nanoparticles [9]. Due to the strong quantum confinement effect of free electrons in the particles, the continuous density of states breaks up into discrete energy levels. These lead to size-dependent fluorescence and other attractive moleculelike properties such as facile surface tailor ability and color tenability [10-13].

As to size-dependent fluorescence, the photophysical properties of AuNCs are dictated by their sizes and shapes. Recent advances make it much easier to synthesize watersoluble AuNCs with tunable size or emission colors. AuNCs 
has an extremely high surface-to-volume ratio, which can make the NCs for further surface modification and controllable bioconjugation. With all the above properties, AuNCs can be used in a wide range of applications, such as biodetection [14], biosensing [14], biological labeling [15-17], and bioimaging [18].

When fluorophores absorb light with a particular wavelength, fluorophores will then emit energy in a form of fluorescence equaling to the energy difference between the excited state and the ground state. Fluorescent probes are the key factor in biological fluorescent measurement. In last decades, there are several different classes of fluorescent labels: organic fluorophores such as cyanine dyes and fluorescein, the rare earth NPs (quantum dots) [19], upconverting NPs, and novel fluorescent dye-doped core-shell nanobeads. But the use of these labels is limited by the poor photostability, large physical size, and toxicity of heavy metals [17]. On the contrary, AuNCs show strong photoluminescence, good photostability and high emission rates, large Stokes shift, and size-dependent tunable fluorescence, for which they can be made as an optimum nanomaterial for biological imaging, analysis, and sensing. Moreover, the surfaces of AuNCs possess a subnanometer dimension, color tenability, facile synthesis, and low toxicity [20,21]. Compared with common fluorophores, these advantages establish them to be a novel ultrasmall fluorophores in biomedicine application [22-24].

In this review, we firstly will discuss the origin of fluorescence of AuNCs. Then we will summarize general synthesis strategies of AuNCs and describe their distinctive properties, which are followed by highlighting their fascinating photoluminescence properties. In the second part, we will discuss the recent advances of AuNCs-based application to the biological labeling and fluorescent imaging. In the final section, we will give a brief outlook on the challenges and prospective for future research of AuNCs.

\section{Gold Nanoclusters: Fluorescence Property and Synthetic Method}

2.1. Fluorescence Mechanism of AuNCs. Gold nanomaterials can be broadly categorized into two kinds according to their dimensions: gold nanoparticles and gold nanoclusters. Zheng has introduced the differences in structures and other properties between these two gold nanomaterials [25]. As he mentioned, gold nanoparticles which sizes are close to the wavelength of light have similar optical properties to bulk gold. What is more, the collective oscillation of conduction electrons on the surface of gold nanoparticles can interact with light and generate SPR effect when the frequency of oscillating electrons matches the frequency of incident photons. And when the gold nanoparticles have a dimension in 3$100 \mathrm{~nm}$, the SPR band locates in the visible light region, which will lead to some size-dependent effects of gold NPs. The interaction between nanoparticles, the dielectric medium, and the protective ligands also has influences on the SPR effect of gold NPs.

When size of gold nanomaterials is smaller than $3 \mathrm{~nm}$, the gold nanoclusters possess some distinguished properties due to their ultrasmall dimension and special structure. Because the energy level spacing is inversely proportional to the radius of AuNCs, AuNCs with a dimension close to Fermi wavelength of an electron possess size-dependent electronic structure [26]. The AuNCs we are discussing in this review are subnanoscale nanoparticles, which have a gold center containing a countable number of gold atoms. So AuNCs are considered as superatoms or molecular-like species. For these reasons, the energy level spacing and free electronic density of AuNCs are the key factors of transition energy of AuNCs. When the energy level spacing is large enough, the free electrons behavior can be observed in the discrete energy levels [27]. According to the structural analysis of Jin's group, the fluorescence of AuNCs originates from the LUMO-HOMO transition [28]. To sum up, the distinct optical properties (absorption and fluorescence) of molecular-like AuNCs are mainly affected by their ultrasmall size and corresponding electronic structure.

In addition to the ultrasmall size, the ligands used for AuNCs preparation also have impacts on their fluorescence properties [5]. Ligands play very important roles in the formation of AuNCs as protective agents, which can prevent the superatoms from aggregation and then keep the sizedependent fluorescence property. As for the demonstration that Jin and coworkers mentioned, surface ligands of AuNCs not only can be used as capping agent but also largely affect the fluorescence of AuNCs by charging transfer from surface ligand to the gold core. When the surface ligands have strong electron donation capability, the fluorescence can be enhanced. And the ligands with electron-rich atoms or groups have been found as a very effective choice for promising surface ligand of AuNCs to enhance the fluorescence. In this paper, we will discuss the synthesis of AuNCs by using different ligands such as glutathione, dendrimers, polymers, and protein; the as-prepared AuNCs based on different synthetic methods have various fluorescence quantum yield (QY). Lots of researches have been carried out on the origin of fluorescence from AuNCs and the enhancement of fluorescence QY for the practical application such as biological labeling, sensing, and imaging. In this section, we have summarized the different fluorescence QYs of AuNCs with different synthetic routes (Table 1).

2.2. Synthetic Methods of AuNCs. Small gold nanoclusters with robust quantum effect have been extensively investigated. The synthesis of AuNCs experiences several stages. In the early time, the research was focused on gas state metal clusters [29]. Since these metal clusters in the gas phase were short-lived and hard to be functionalized, a novel method, solution-phase synthesis, arose in the 1980s [30, 31]. These synthetic strategies lead to gold nanoclusters with enhanced stability and excellent physicochemical properties.

Since Brust et al. reported the synthesis of monolayer protected metal nanoparticles, several novel advances have emerged in the field of metal nanoclusters, especially in the field of thiol-containing AuNCs. During the decades, the synthetic strategies were generally considered as two routes: "Atoms to Clusters" and "Nanoparticles to Clusters." The approach of "Atoms to Clusters" is to reduce the gold ions into zerovalent atoms, and then AuNCs are formed with the nucleation of the Au atoms. However, the gold precursors 
TABLE 1: Summary of fluorescence QYs of AuNCs obtained by different synthetic approaches.

\begin{tabular}{|c|c|c|}
\hline Synthesis & Fluorescence QYs & References \\
\hline Protected by monolayers of glutathione & $(3.5 \pm 1.0) \times 10^{-3}$ & [32] \\
\hline Alkanethiol ligands 11-mercaptoundecanoic acid (11-MUA) as stabilizer & $3.1 \times 10^{-2}$ & {$[33]$} \\
\hline Poly(amidoamine) (PAMAM) dendrimers as stabilizer and capping agent & $41 \pm 5 \%$ & {$[34]$} \\
\hline $\begin{array}{l}\text { Pentaerythritol tetrakis(3-mercaptopropionate)-terminated Polymethacrylic acid } \\
\text { (PTMP-pMAA) as capping agent }\end{array}$ & $3 \%$ & {$[35]$} \\
\hline Employed poly(N-vinylpyrrolidone) (PVP) as stabilizer of the gold clusters & $12.5 \%$ & {$[36]$} \\
\hline Bovine serum albumin molecules as templates and reductants & $\sim 6 \%$ & [37] \\
\hline Bovine pancreatic ribonuclease A (RNase-A) as the biotemplate & $\sim 12 \%$ & {$[38]$} \\
\hline $\begin{array}{l}\text { DNA as template to prepare gold/silver nanoclusters from } \mathrm{Au}^{3+}, \mathrm{Ag}^{+} \text {, and DNA } \\
\left(5^{\prime} \text {-CCCTTAATCCCC- } 3^{\prime}\right)\end{array}$ & $4.5 \%$ & [39] \\
\hline Employing L-3,4-dihydroxyphenylalanine (L-DOPA) as a reducing/capping reagent & $1.7 \%$ & {$[40]$} \\
\hline D-penicillamine as capping agent & $1.3 \pm 0.3 \%$ & {$[41]$} \\
\hline Mercapto-9-propyladenine as capping agent & $1.2 \%$ & {$[42]$} \\
\hline$N, N$-dimethylformamide as a weak reducing agent as well as stabilizing ligand & $14.28 \%$ & {$[43]$} \\
\hline Dissociation process of glutathione-gold(I) polymers in aqueous solution & $4.0 \pm 0.4 \%$ & {$[44]$} \\
\hline $\begin{array}{l}\mathrm{GSH} \text { as reductant to form } \mathrm{Au}(\mathrm{I}) \text {-thiolate complexes, and the aggregation of } \\
\mathrm{Au}(\mathrm{I}) \text {-thiolate complexes on the } \mathrm{Au}(0) \text { to form } \mathrm{Au}(0) @ \mathrm{Au}(\mathrm{I}) \text {-thiolate NCs }\end{array}$ & $\sim 15 \%$ & {$[45]$} \\
\hline $\begin{array}{l}\text { 11-Mercaptoundecanoic acid (11-MUA) as the protecting group to reduce } \mathrm{Au}^{3+} \text { with } \\
\mathrm{NaBH}_{4} \text { in methanol solutions }\end{array}$ & $\sim 6.92 \%$ & {$[46]$} \\
\hline $\begin{array}{l}\text { Polyethylene glycol (PEG) appended with lipoic acid (LA) anchoring groups as } \\
\text { modular ligands }\end{array}$ & $14 \%$ & {$[47]$} \\
\hline
\end{tabular}

can be reduced into gold nanoparticles easier than into nanoclusters due to the possibility of AuNCs to aggregate. In order to solve the problem, some kinds of ligands should be used for surface modification. Moreover, the protective ligand capped on the surface can enhance the fluorescent effect. These show that choosing appropriate ligands is the key factor in stabilizing cluster from aggregation. The other route for synthesizing the AuNCs is "Nanoparticles to Clusters" route. Pradeep and coworker used excess MSA ligands in toluene to etch (MSA-) protected large Ag nanoparticles into fluorescent Ag NCs [48]. Recently, some advances have been reported [49-51] to form more stable, monodisperse, and highly fluorescent AuNCs.

\subsection{1. "Atoms to Clusters" Route}

(1) Template-Based Synthesis Methods. AuNCs with ultrafine size and nontoxicity are very attractive for biolabeling and bioimaging application. For the reason of low fluorescent QY $\left(10^{-4}-10^{-1} \%\right)$ of thiol-protected AuNCs, Dickson's group developed NCs exhibiting $\sim 40 \%$ QY by dendrimers-assisted method in the early 2000s [34]. This synthesis can effectively enhance the fluorescent effect of AuNCs with a fairly long reaction time. According to the first template-assisted techniques, a series of templates such as polymers $[35,52]$, proteins [53, 54], dendrimers [55], and DNA [56, 57] have been investigated for the synthetic method of AuNCs. Compared with other synthetic techniques, template-protected techniques use mild reductant in the presence of strong stabilizer. The decreased clustering rate provides efficient sequestering by stabilizers. (i) Dendrimers. Dendrimers-encapsulated AuNCs exhibited strong fluorescence in the range from blue to NIR region by adjusting the generation of PAMAM and the ratio between gold and the template [55]. Water-soluble, monodisperse, highly fluorescent $\mathrm{Au}_{8} \mathrm{NCs}$ had been synthesized by using the fourth generation of polyamidoamine (G4-OH PAMAM) dendrimers as reduction agent and stabilizer [58, 59] by Dickson and coworkers in the early 2000s [34, 55]. The asprepared AuNCs exhibit a strong fluorescence (QY 40\%), with an intensity of 100-fold higher than AuNCs prepared by other methods, possessing strong blue luminescence with excitation and emission peak at $385 \mathrm{~nm}$ and $450 \mathrm{~nm}$, respectively, shown in Figure 1.

Using charged PAMAM dendrimers of generation 6-9 to prepare size-tunable fluorescent AuNCs in water have been reported. The production have been characterized by a set of methods such as tranmission electron microscopy (TEM) to demonstrate gold atoms encapsulated by dendrimers. In 2007, Bao et al. unveiled a new method for synthesizing nontoxicity, highly stable AuNCs by using a mild reduction agent-ascorbic acid or no reductant at physiological temperature [60]. This approach produced highly green and red emission AuNCs without forming large gold nanoparticles. Lin and coworkers [61] exploited an amphipathic microcavity-template for sequestering gold ions and synthesizing AuNCs with high QY (20-60\%) through microwave irradiation. In this strategy, the fourth generation PAMAM with amine-terminated groups (G4NH2) had a more hydrophilic microcavity than hydroxyl-terminated G4OH PAMAM, which led to the polarity-dependent ion-pair association $\left(\mathrm{AuCl}_{4} / \mathrm{G}_{4} \mathrm{NH}_{2}\right.$ pair and $\mathrm{AuBr}_{4} / \mathrm{G} 4 \mathrm{OH}$ pair). The strong ion-pair association was 


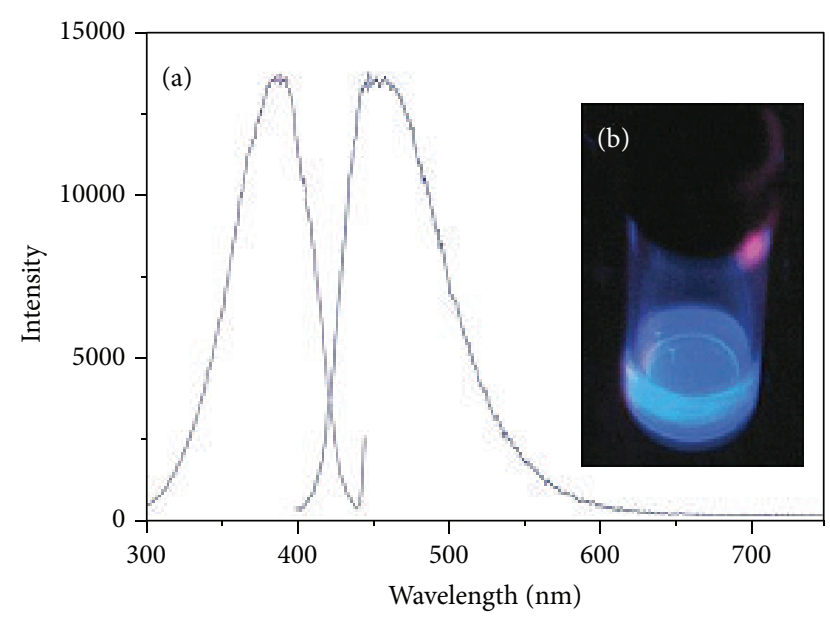

FIgure 1: (a) Excitation and emission spectra of G4-OH PAMAMtemplated AuNCs. (b) Emission from AuNCs under long-wavelength UV lamp irradiation $(366 \mathrm{~nm})$. Reprinted with permission from [34], ( 2003 , American Chemical Society.

capable of enhancing the quantum efficiency of AuNCs, and the precise control of the reaction temperature assisted by microwave irradiation could also lead to the same effects.

(ii) Polymers. Polymers are widespread macromolecule with abundant functional groups, which have potential to be templates for synthesizing AuNCs [62, 63]. Using polymers to prepare AuNCs has been studied by several groups. For example, AuNCs protected by multivalent polymers were synthesized by ligand exchange with dodecylamine-capped gold nanoparticles [64]. The ligand-induced etching method leads to fluorescent AuNCs with a relatively low QY (10$20 \%)$. The size of polymer-depended AuNCs is related to several factors such as the structure of polymer, the nature of polymer functional group, and the ratio of gold to polymer $[35,65,66]$. A switch over nonfluorescent to fluorescent polymer-capped AuNCs (core diameters: $1.1-1.7 \mathrm{~nm}$ ) can be finely tuned by adjusting the ratio of gold-to-polymer as reported by Cooper et al. Moreover, González et al. have discovered a new simple method for preparing AuNCs protected by poly(N-vinylpyrrolidone) (PVP), which possess the fluorescent and magnetic features [36]. In this method, an electrochemical synthesis has been used to pursue a much simpler and quicker synthetic route. Most recently, Jiang and coworkers have developed novel hybrid nanogels based on the NIR fluorescent AuNCs and poly(acrylic acid) (PAA) [67]. The core-hollow and shell-porous PAA nanogels were employed as templates followed by in situ reduction of $\mathrm{HAuCl}_{4}$ in water, to synthesize AuNCs-encapsulated PPA hybrid nanogels. The PAA nanogels were prepared by the polymerization of acrylic acid monomer and then mixing the products with cysteamine water solution through interaction of $\mathrm{COO}^{-}$group of PAA and $\mathrm{NH}_{2}{ }^{+}$of cysteamine. $\mathrm{Au}^{3+}$ ions were subsequently added and reduced by thiols of cysteamine so as to encapsulate $\mathrm{Au}^{+}$into the cavities of PAA nanogels. Finally, $\mathrm{NaBH}_{4}$ was chosen to reduce the Au ions into AuNCs, shown in Figure 2. This AuNCs entrapped in PAA nanogels

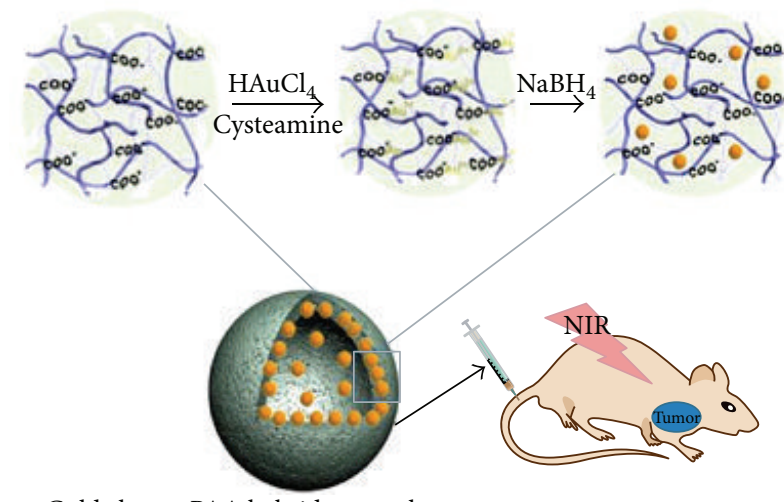

Gold cluster-PAA hybrid nanogels

FIGURE 2: Schematic of synthetic method of gold cluster-encapsulated PAA hybrid nanogels. Reprinted with permission from [67], (C)2013, American Chemical Society.

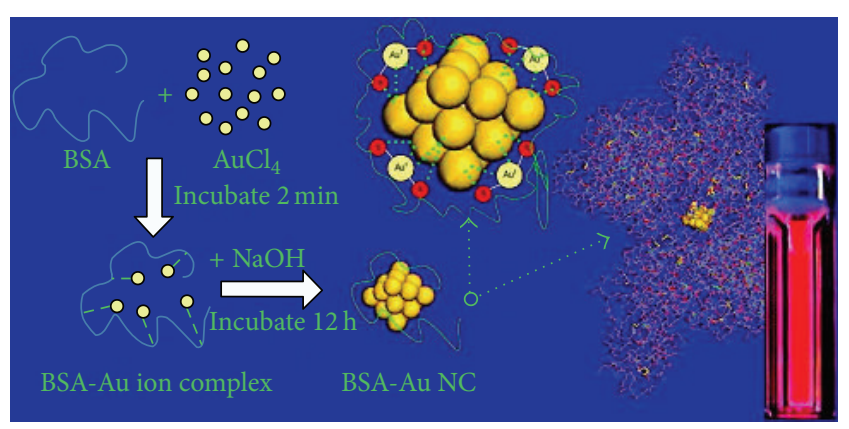

FIGURE 3: Schematic of the formation of BSA-templated AuNCs. Reprinted with permission from [37], (C2009, American Chemical Society.

possessed the NIR fluorescent property with deep tissue penetration, which was suitable for NIR live imaging.

(iii) Proteins. Proteins, as biological macromolecules, have certain configuration and spaces and can be utilized as templates for preparing fluorescent AuNCs. Xie and coworkers have developed a class "green" synthesize method to prepare protein-directed AuNCs [37]. In this method, a facile, onepot, "green" synthetic technique was developed based on a common commercially available protein, bovine serum albumin (BSA), as shown in Figure 3.

BSA, a plasma protein existing in abundance, was selected as the model protein for the formation of AuNCs because of its stability, lack of effect, and low cost. Similar to the biomineralization behavior in nature, the $\mathrm{Au}^{3+}$ ions were added to the aqueous BSA solution, to be reduced to $\mathrm{Au}^{+}$with the help of tyrosine residues on the protein, and then metal ions were sequestered and entrapped by thiol groups of the protein. The as-prepared AuNCs were stable at the physiological temperature and $\mathrm{PH}$ with a QY 6\%. The as-synthesized BSA-AuNCs have 25 gold atoms in the core, which exhibit red emission (maximum of emission wavelength locates in $640 \mathrm{~nm}$ ), excellent stability, good biocompatibility, and easy surface modification. This striking work is not only 
a novel method for creating highly fluorescent AuNCs, but a promising protocol can be used for the other proteins and noble metals. Following to this report, many groups studied this "green" synthetic route with other bioactive proteins such as insulin [68-70], lysozyme [71], transferrin [72], and egg white proteins [53]. Chou et al. have firstly selected insulin as the template for synthesizing the fluorescent AuNCs. The asprepared insulin-AuNCs are applicable for fluorescent imaging, CT, and in vivo blood-glucose regulation. As innovative imaging technique involving insulin, it should be extensively studied in biolabeling and bioimaging application. The use of BSA as a reductant and stabilizer has enlightened Lu et al. to find some proteins expect BSA for preparing AuNCs. They have discovered a way to prepare highly fluorescent AuNCs by using lysozyme as reductant and stabilizer. The as-synthesized lysozyme gold fluorescent NCs (LsGFC) have a diameter of $\sim 1 \mathrm{~nm}$ and emission wavelength centered at $657 \mathrm{~nm}$, which also can be specifically quenched by $\mathrm{Hg}^{2+}$. Very recently, chicken egg white (CEW), which is abundant for being available and extremely cheap, has been exploited as a template for synthesizing AuNCs by Geckerler et al. In the method, researchers developed a green, rapid synthesis of AuNCs using CEW at physiological temperature $\left(37^{\circ} \mathrm{C}\right)$ with $\mathrm{pH} 11$. Under alkaline conditions, tyrosine and tryptophan are predominantly responsible for reduction of $\mathrm{Au}^{3+}$ ions and obtained highly fluorescent, water-soluble AuNCs. Most recently, Qiao and coworkers have exploited a novel fluorescent nanoprobe which contains fluorescent ovalbumincapped AuNCs, biotarget folic acid, and homopolymers as the linker [72]. Unlike most polymer-capped nanoclusters, folic acid-functionalized fluorescent AuNCs can specificly label the cell and tumor, which have superiority in biolabeling and bioimaging applications.

(iv) DNA. The introduction of biological macromolecules (protein, DNA) can make the synthetic method of AuNCs greener and more biocompatible, which renders them more possible for biological application. Some reports have been published to make the affinity of DNA base pair and AuNCs evident theoretically $[73,74]$, which provide the foundation for the subsequent experiments to verify the feasibility of theories. Chen and coworkers have unveiled atomically monodispersed AuNCs with direction of DNA as etchant of gold nanoparticles and rods [75]. Liu and colleagues firstly reported that $\mathrm{pH}$-dependent single-stranded DNA (ssDNA) was feasible in synthesizing blue-emitting AuNCs with a mild reductant [76]. Shao and colleagues developed a nanoparticle-free method for synthesizing red-emitting AuNCs in water with DNA as a template [77].

(v) Small Molecules. Most kinds of AuNCs are synthesized by exploiting large templates such as dendrimers, biological macromolecules (e.g. DNA, proteins) and polymers. Meanwhile, small molecules used as the templates have been paid much attention for synthesizing high fluorescent AuNCs, especially the thiol-protected (glutathione, 11-mercaptoundecanoic acid) AuNCs [79]. However, using small molecules as the templates and reductants has not drawn that much attention for synthesizing high fluorescent AuNCs compared with other templates. Several small molecules

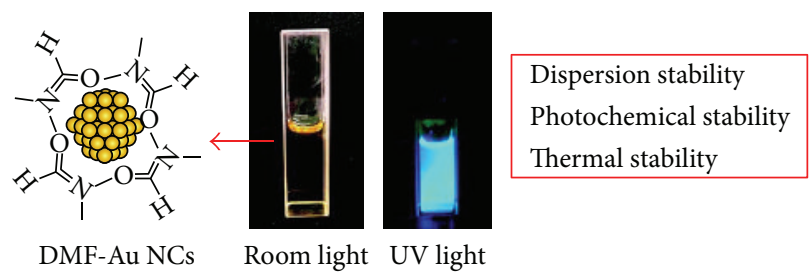

(a)

(b)

FIgURE 4: Schematic of DMF-AuNCs construction (a); the photographs of DMF-protected Au clusters in DMF under ambient light (light yellow) and UV light of $365 \mathrm{~nm}$ (blue) (b). Reprinted with permission from [78], (c2010, Langmuir.

have been exploited in the fabrication of AuNCs such as amino acid [80-83], peptide [84, 85], dimethylformamide $[43,78]$, and Good's buffers [86]. The report using amino acid as a reactant for preparing AuNCs is scarce. Chen and coworkers [83] have discovered a fast, facile method for preparing AuNCs by blending Chloroauric Acid $\left(\mathrm{HAuCl}_{4}\right)$ with histidine. It is the first time for preparing AuNCs with amino acid as the reductant and stabilizer at the same time. Kawasaki et al. have reported the DMF-protected AuNCs through the interactions of the amide groups of DMF with AuNCs, which possess high thermal stability, high dispersion stability in various solvents, and high photochemical stability, as shown in Figure 4 [78].

2.2.2. Ligand-Protected Gold Nanoclusters. Early studies for the AuNCs are focused on synthesizing by strong reducing agent and strong capping agents such as thiol [87, 88], dendrimers [34], and polymers [89]. For synthesizing high yield of AuNCs, a practical way was discovered involving weaker reducing agent in the present of strong capping agent. This method can form nanoparticle-free AuNCs efficiently with slower reduction rate [44]. Due to excellent optical, electronic, and chemical properties, ligand-protected gold nanoclusters have been extensively investigated, especially for their potential applications in biomedicine and nanoelectronics.

(1) Phosphine as Ligand [91]. In the infant stage, phosphine molecules have been widely utilized as a ligand during the preparation of AuNCs [92, 93]. The phosphine-capped AuNCs are stable to the ambient conditions and controllable over cluster core size. Generally, phosphine-capped AuNCs are synthesized by sodium borohydride reduction. In the 1960s, an 11 gold atoms phosphine-protected cluster, $\mathrm{Au}_{11}\left(\mathrm{PPh}_{3}\right)_{7}(\mathrm{SCN})_{3}$, was firstly reported [92]. And an $\mathrm{Au}_{13}{ }^{-}$ centered icosahedral structure cluster was successfully prepared in 1981 [94]. Schmid et al. reported $\mathrm{Au}_{55}$-centered nanoclusters with an average core size of $\sim 1.4 \mathrm{~nm}$ [95]. The method was optimized by Hutchison and coworkers, who developed a more convenient, safer approach to synthesize phosphinecapped gold nanoclusters [96]. In recent development of phosphine-capped gold nanoclusters, Wan et al. isolated new phosphine-protected $\mathrm{Au}_{20}$ nanoclusters through the reduction of $\mathrm{Au}\left(\mathrm{PPhpy}_{2}\right) \mathrm{Cl}\left[\mathrm{PPhpy}_{2}=\right.$ bis (2-pyridyl) phenylphosphine] by $\mathrm{NaBH}_{4}$, the $\mathrm{Au}_{20}$ core was the fusion of 


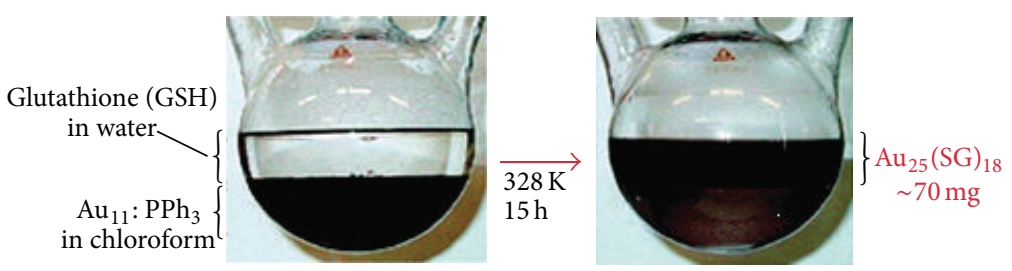

FIGURE 5: Phosphine-stabilized $\mathrm{Au}_{11}$ clusters in chloroform were reacted with glutathione (GSH) in water under a nitrogen atmosphere. Reprinted with permission from [90], (C2005, American Chemical Society.

two $\mathrm{Au}_{11}$ clusters [97]. Although phosphine-capped AuNCs are one of the important classes in the gold clusters families, the synthetic method still faces some problems such as tedious extractions, numerous steps, and unstable production tending to decompose. Since gold atoms have a good affinity to $-\mathrm{SH}$ groups, the unstable phosphine-stabilized AuNCs could be transferred into thiol-stabilized gold clusters. Hutchison and colleagues have reported a versatile ligandexchange reaction and first described the mechanism of thiolphosphine ligand exchanges [98]. As shown in Figure 5, Shichibu et al. synthesized glutathione-capped AuNCs $\left(\mathrm{Au}_{25}\right.$ $(\mathrm{SG})_{18}$ ) by ligand exchange with phosphine-stabilized $\mathrm{Au}_{11}$ clusters. Teranishi and coworkers recently reported a bulk solution synthetic method that permitted large scale, facile synthesis of truly monodisperse $\mathrm{Au}_{38}$ nanoclusters, which used a two-phase ligand exchange process based on GSH-Au clusters, and led to monodisperse $\mathrm{Au}_{38}$ nanoclusters in high purity [90].

(2) Thiolate-Capped Gold Nanoclusters. Brust et al. have done the pioneering work based on the reduction of the metal precursors and the formation of metal core [99]. Owing to a strong affinity for thiols and $\mathrm{Au}$, thiol-containing small molecules were extensively used to stabilize gold nanoclusters in the aqueous solution [6]. Using thiol-containing small molecules as stabilizers rendered much more controllable AuNCs than phosphine-capped ones, contributing to the stronger Au-S covalent bonding. Thus they have been intensely pursued in last decades.

Generally, the method of synthesizing thiolate-capped AuNCs has processes as follows. Gold salts $\left[\mathrm{AuCl}_{4}\right]^{-}$are dissolved in water and then transferred to an organic solvent by phase transfer agent; the thiols are added to the mixture to reduce $\mathrm{Au}^{3+}$ ions into $\mathrm{Au}^{+}$ions and form $\mathrm{Au}^{+}-\mathrm{SR}$ complexes or polymers; then the $\mathrm{Au}^{+}$polymers are reduced by adding the reducing agent into thiolate-protective gold nanoclusters.

The fluorescence of thiolate-protected AuNCs (referred to as $\mathrm{Au}_{n}(\mathrm{SR})_{m}$, where $n$ and $m$ are the respective number of metal atoms and ligands) was effected by the kind of ligand and core charge state. In order to enhance fluorescence, different chain lengths of thiols will be chosen for the biolabeling and imaging applications [100]. Jin and colleagues discovered "size focusing" method to synthesize atomically monodisperse thiolate-stabilized AuNCs using different thiol ligand (-SR: $-\mathrm{SC}_{2} \mathrm{H}_{4} \mathrm{Ph},-\mathrm{SC}_{12} \mathrm{H}_{25}$, SG, and $\mathrm{SC}_{10} \mathrm{H}_{22} \mathrm{COOH}$ ) [101].

Glutathione (GSH), a ubiquitous low-molecular weight thiol, played a significant role in making AuNCs which showed good water solubility, bioactive surface, and high stability. It has been widely investigated for protecting the $\mathrm{Au}^{3+}$ ions when they were being reduced by sodium borohydride $\left(\mathrm{NaBH}_{4}\right)$ [102-104]. Whetten and coworkers have unveiled an unprecedented thiol-protective AuNCs by using the GSH (N- $\gamma$-glutamyl-cysteinyl-glycine) as the stabilizer. The as-synthesized AuNCs were fractionated by using polyacrylamide gel electrophoresis (PAGE) and characterized by mass spectrometry (MS) [102]. Tsukuda and colleagues have also reported the characterization of fractionated AuNCs protected by GSH monolayers using mass spectrometric. The as-prepared AuNCs were isolated into single-sized $\mathrm{Au}_{n}(\mathrm{SR})_{m}$ clusters by the PAGE method with $n=18,21,25,28,32,39$ (called magic-numbered clusters) [103]. Liu and coworkers employed GSH-stabilized AuNCs (GSH-Au NCs) as the sensor of $\mathrm{Cr}$ (III) and $\mathrm{Cr}$ (VI) through fluorescence quenching [105]. In the method, GSH was used as an environment friendly reducing/protecting regent for preparing AuNCs in physiological temperature for $24 \mathrm{~h}$. The as-prepared AuNCs exhibited yellow under visible light, but the color turned into bright red when radiated by UV light, as shown in Figure 6 .

Moreover, various other thiols can be used to stabilize the AuNCs such as tiopronin [106], phenylethylthiolate [107], thiolate $\alpha$-Cyclodextrin $[108,109]$, mercaptopropionic acid [110, 111], bidentate dihydrolipoic (DHLA) [112], dodecanethiol [113], and D-penicillamine $[41,114,115]$. Using these mercaptolinkers above makes the AuNCs possess higher fluorescent yield and monodispersion. Mattoussi and coworkers [47] prepared a highly fluorescent gold nanoclusters with emission centered at $\sim 750 \mathrm{~nm}$ NIR region using bidentate ligand as stabilizing agent. Shang et al. have reported a novel watersoluble Au-SR using the D-penicillamine (DPA) and a mild reductant. The DPA-capped AuNCs show an enhancement of the fluorescence intensity and a remarkable stability $(\sim \mathrm{QY}=$ $1.3 \%)$, which have potential for bioimaging application.

2.3. "Nanoparticles to Clusters" Route. With the development of techniques, a novel method is reported that involves etching surface atoms of gold nanoparticles by appropriate ligands. Different small molecules and polymers have been exploited as the etchant such as thiols (e.g., glutathione, dihydrolipoic acid, and phenylethylthiol) $[16,116,117]$ and biomacromolecules (e.g., BSA). Typical procedure contains the following: first, mixing the surface-stabled gold nanoparticles with excessive etchant (small molecules/polymers) and then etching the gold NPs' surface via ligand exchange and finally, generating etchant-gold complexation. Using this method can enhance the fluorescent effect of AuNCs (QY 4$20 \%)$. 


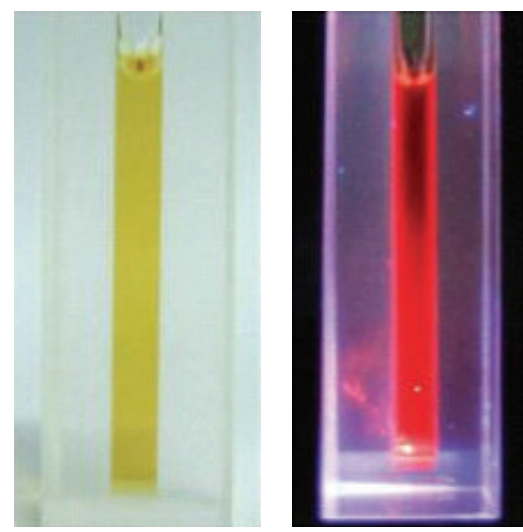

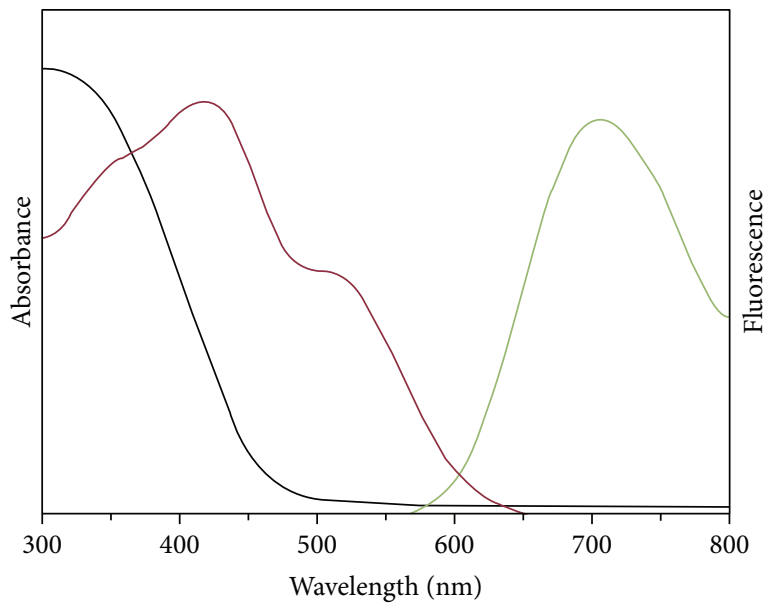

- Absorption

- Excitation

(a)

(b)

Figure 6: (a) GSH-protected AuNCs under visible light (left) and UV light (right), respectively. (b) UV/vis absorption, fluorescence excitation (emission at $710 \mathrm{~nm}$ ), and emission (excitation at $410 \mathrm{~nm}$ ) spectra of GSH-Au NCs. Reprinted with permission from [105], (2013, Science Direction.

2.3.1. Ligand-Induced Etching. In the past decades, a series of protecting ligands have been exploited to prepare relatively controllable monolayer protected clusters (MPCs). The ligands such as phosphines, thiols, amines, and polymers have multiple functions in effecting the cluster size and physical/ chemical properties. Besides the one-pot method by direct reduction of $\mathrm{Au}^{3+}$ ions in the presence of thiols mentioned above, fluorescent gold MPCs can be synthesized by etching Au core with excessive ligands. The study of monolayer gold MPCs has been separated into three stages: polydispersed nanoclusters; monodispersed nanoclusters; and atomically precise nanoclusters [118]. Jin et al. have discovered a kinetically controlled method based on ligand-induced etching for preparing atomically monodisperse Au-SR, called "size focusing" method. For instance, Jin and coworkers developed a facile, high yielding synthetic approach to synthesize $\mathrm{Au}_{38}$ $\left(\mathrm{SC}_{2} \mathrm{H}_{4} \mathrm{Ph}\right)_{24}$ clusters with excess phenylethylthiol. Schaaff and coworkers conducted thiol etching on a combination of $14 \mathrm{k}$ and $8 \mathrm{k}$ species, the result showed that the $8 \mathrm{kDa}$ species became enriched [119]. Duan and colleague have reported synthesizing blue-emitting monodisperse AuNCs by exploiting the etching property of hyperbranched and multivalent polymers-polyethylenimine (PEI). In the approach, asprepared $\mathrm{Au}$ nanocrystals in dimension for $\sim 8 \mathrm{~nm}$ were synthesized by modified Brust-Schiffrin method. Once acting with excessive PEI, the separated AuNCs with 8 atoms emitted intense green light under UV light irradiation (365 nm), as shown in Figure 7 [64].

Parak and coworkers reported a precursor-induced etching method for the preparation of water-soluble fluorescent dihydrolipoic acid-capped AuNCs [16]. In the method, gold NPs stabled by didodecyldimethylammonium bromide (DDAB) were synthesized in the organic phase, followed by precursor induced etching into AuNCs. Synthesis of watersoluble AuNCs was depending on the ligand exchange with reduced lipoic acid (DHLA). Upon the production transfer to aqueous solution, they became intensely red-luminescent with QY 3.45\%. This report exhibited a totally novel method for preparing ultrasmall fluorescent AuNCs even if the detailed mechanisms have not yet been clearly elucidated.

2.3.2. Solvent-Induced Etching. Phase transfer is demonstrated to be an effective approach for preparing the fluorescent metal NCs via the electrostatic interaction. Yuan et al. [51] have developed a facile and versatile synthesis of highly fluorescent, stable, and monodispersed AuNCs based on a mild etching of originally polydispersed, nonfluorescent, and unstable gold nanocrystals. Thiol-protected AuNCs were synthesized by reducing the $\mathrm{Au}^{+}$-SR with the reducing agent $\left(\mathrm{NaBH}_{4}\right)$ in the presence of glutathione $(\mathrm{GSH})$ and then transferring the polydispersed thiol-protected AuNCs into an organic phase by adding cetyltrimethylammonium bromide (CTAB) due to the electrostatic interactions between negatively charged carboxyl group in GSH and the positively charged cations of the hydrophobic salt $\left[(\mathrm{CTA})^{+}(\mathrm{COO})^{-}\right]$. The mild etching happened in the organic phase which led to enhanced fluorescent monodisperse AuNCs. Moreover, the fluorescent AuNCs can easily be transferred back to the aqueous solution by injecting a salt to remove the (CTA) ${ }^{+}$. This approach is used to fabricate highly fluorescent AuNCs with excellent stability, biocompatible protecting ligands, and monodispersion, which has a promising prospect in bioimaging and biosensing application. Xie and coworkers have also discovered a novel Au-thiolate NCs with highly luminescence $(\mathrm{QY} \sim 15 \%)$. The mechanism of aggregation-induced emission (AIE) has been discovered. Due to the discovery, 


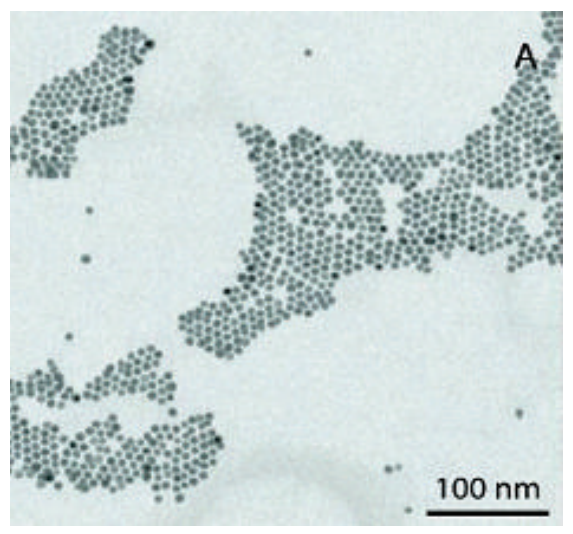

(a)

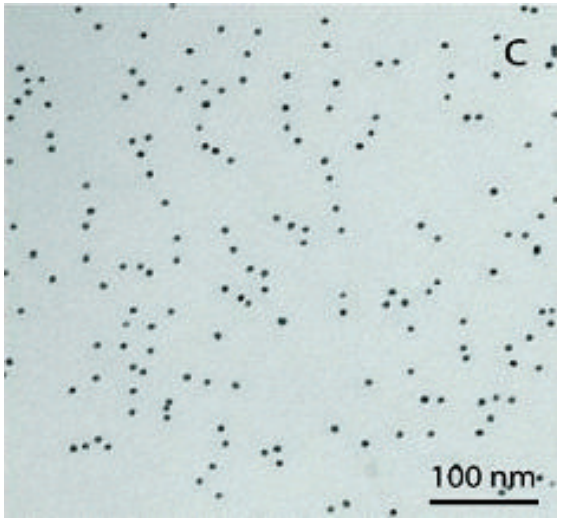

(c)

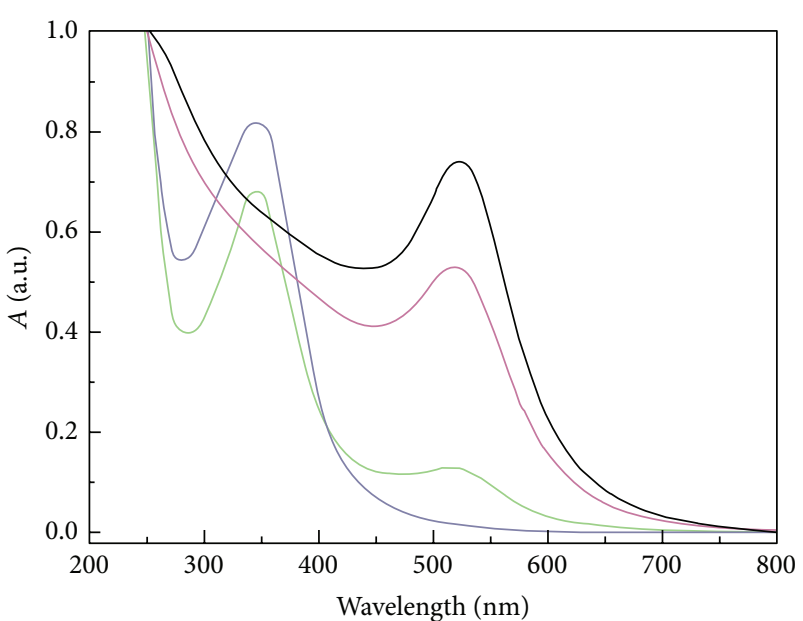

(b)
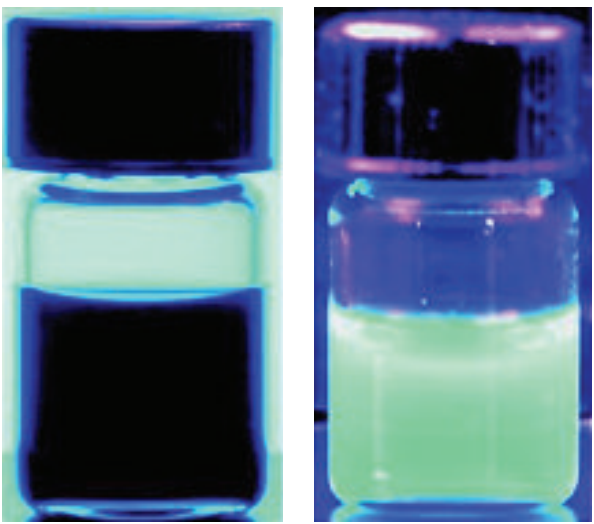

(d)

FIGURE 7: (a) and (c) are TEM of Au nanocrystals before and after ligand-induced etching. (b) UV-vis spectra of the original Au nanocrystals (black), the etched nanocrystals after separation (red), the AuNCs mixture after etching (green), and the purified AuNCs after separation (blue). (d) Color photographs of the original Au nanocrystals in chloroform (left) and the purified AuNCs in water (right). UV light (365 nm). Reprinted with permission from [64], (2)(2007), American Chemical Society.

the nonfluorescent oligomeric Au (I)-thiolate complexes can generate strong luminescent under UV light depending on the degree of aggregation. The AIE properties can be used for designing AuNCs formed by the aggregation of $\mathrm{Au}$ (I)thiolate complexes and in situ generated Au core, which aggregation was induced by adding a high concentration of ethanol in water, as shown in Figure 8 [45].

\section{Application of Gold Nanoclusters: Biosensing, Biolabeling, and Bioimaging}

AuNCs as nanoprobes have several advantages, as follows. AuNCs have good stability and monodispersion in the physiological environment leading to enhanced sensitivity and increased tracking lifetime; AuNCs possess ultrasmall size which can be easily uptaken by cells; the biological functions of bioentities conjugated with AuNCs will not be disturbed; photoluminescence in the form of fluorescence is an important property of AuNCs; with decreasing the core size, their fluorescence will show a blue-shift from the NIR region to ultraviolet; the QY of AuNCs is much higher than bulk gold and gold NPs in magnitude; compared with the harsh synthetic steps and tedious surface modification of quantum dots, it is much more achievable to prepare AuNCs and the functional groups induced by the templates and stabilizers are much convenient for coupling with the dyes, peptide, DNA, and other markers; owing to the low content of metal, AuNCs have good biocompatibility for lots of in vitro and in vivo applications.

As mentioned above, fluorescent AuNCs are promising material as fluorescent biolabels and light-emitting sources in nanoscale; therefore, they can be applied in biological labeling, imaging, detection, and so on.

The precondition for making the applications possible is appropriate surface modification for AuNCs, which can eventually influence their stability, biocompatibility, targeting, and other properties. The method functionalizing AuNCs involves surface chemistry and bioconjugation, which should promise AuNCs survival in these conditions. Herein, we will summarize the strategies which are widespread in synthesizing functional AuNCs as fluorescent biological probes in biolabeling, bioimaging, and biosensing applications. 


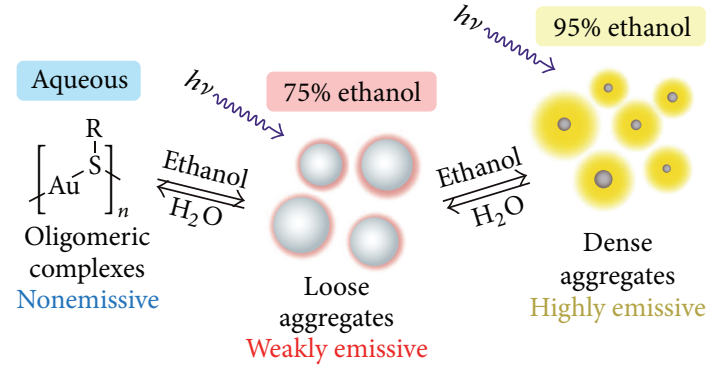

(a)

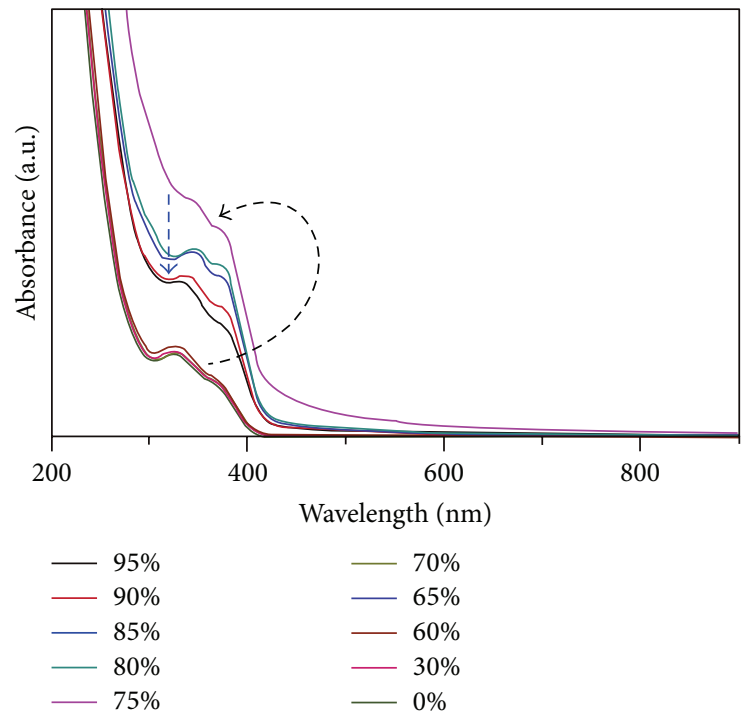

(c)

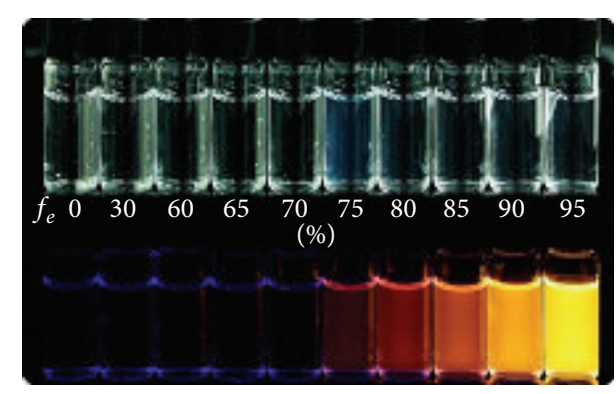

(b)

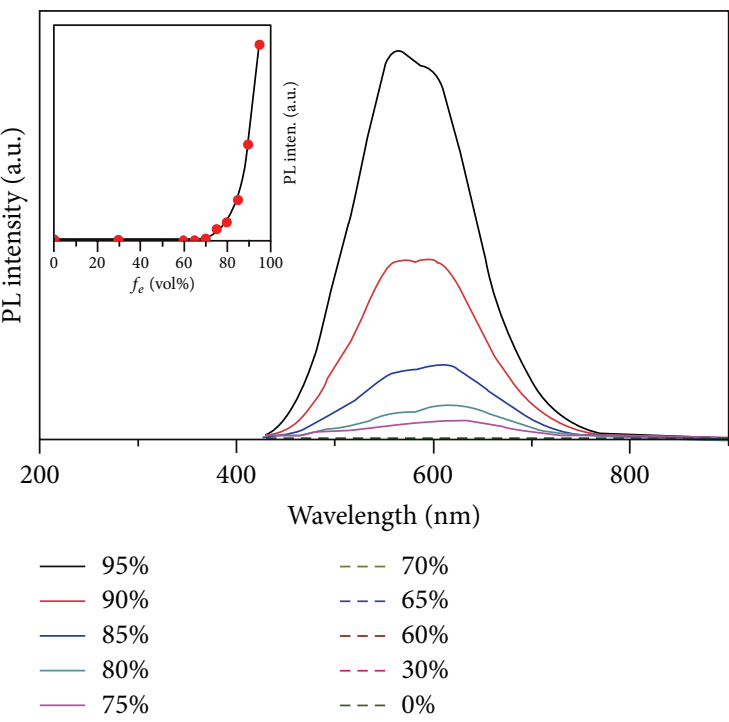

(d)

Figure 8: (a) Schematic illustration of solvent-induced AIE properties of oligomeric Au (I)-thiolate complexes. (b) Digital photos of Au (I)thiolate complexes in mixed solvents of ethanol and water with different $f_{e}$ under visible (top row) and UV (bottom row) light. (c) UV-vis absorption and (d) photoemission spectra of $\mathrm{Au}(\mathrm{I})$-thiolate complexes in mixed solvents with different $f_{e}$. (Inset) Relationship between the luminescence intensity and $f_{e}$. The spectra were recorded $30 \mathrm{~min}$ after the sample preparation. Reprinted with permission from [45], $\odot(2012)$, American Chemical Society.

3.1. Surface Functionalization. Nanoparticles are usually modified with a variety of materials such as silica, synthetic polymers, biopolymers, dendrimers, and small molecules leading to introducing desired functionality $[120,121]$. In the early days, surface modification is based on chemisorption through which bonds are weak and easy to rupture. A novel strategy offering a stronger and more robust bond has been discovered to modify NPs with more stable ligands [122], which make it possible for AuNCs holding promising applications in the biological field, in vitro and in vivo. Hence, there are several demands to AuNCs: (1) they should be watersoluble and stable in a physiological environment; (2) they should possess targeting to specific tissue with high selectivity in vivo; and (3) they should be efficiently discharged by the metabolic system for lowest toxicity [121]. In order to fit the bills, a mountain of work has been done to tailor functional AuNCs with various coating and bioconjugation.

The silica coating, one of the most multifunctional methods of surface functionalization, can couple the advantage of BSA-templated AuNCs with the silica particles. Guével and coworkers [123] have highlighted a novel kind of fluorescent core-shell nanoparticles by BSA-templated AuNCs. The asprepared AuNCs doped Si NPs show monodispersion, higher stability, NIR emission wavelength, and enhanced fluorescent intensity. Moreover, the silica shell allows AuNCs to be conjugated with bioactive entities (e.g., peptide, folic acid (FA), antibody, and polymer).

Bioconjugation is an effective route to introduce extra functionality onto nanoclusters through several functional groups such as primary amide, carboxylic acid, alcohols, and thiols [124]. According to the synthesis of AuNCs as we have discussed before, the majority of template-directed and ligand-protected clusters possess the carboxylic acids on the surface as functional groups, which can conjugate with amino-terminal biomolecules. The reaction is commonly catalyzed with 1-ethyl-3-(3-dimethylaminopropyl) carbodiimide (EDC) and sulfo-N-hydroxysuccinimide (sulfo-NHS) to form amide bond. Yan and colleagues have developed an approach to conjugate Cyclo(Arg-Gly-Asp-D-Phe-Lys) (RGD) peptide to BSA-stabilized AuNCs with the use of coupling agents [125]. A simple one-pot method has been studied by Shang and colleagues for synthesizing D-penicillaminecapped AuNCs, which have both amino and carboxylic 
groups on the surface for further bioconjugation. Abad and coworkers have synthesized AuNCs capped with dihydrolipoic acid (DHLA), which possess hydrophilic carboxyl group providing an anchor for biological molecules conjugation such as covalent linkage [126]. AuNCs coated with carboxylic acid have been bonded with polymers containing amine functionalities by ion pairing without EDC and NHS. Mattoussi and coworkers have reported preparing the AuNCs stabilized with zwitterion-appended lipoic acid ligands, in which process reactive groups (amine or carboxyl group) can be inserted in situ on the surface of production for further bioconjugation [47].

In addition to the further steps for surface functionalization, the introduction of ultrabiocompatible interface and the protection of AuNCs have occurred simultaneously in terms of the template-directed fluorescent AuNCs. For instant, proteins such as BSA are extensively exploited as the stabilizers for preparing fluorescent AuNCs bringing in controllable functional groups $[127,128]$.

3.2. Biological Sensing Application. The minitype sensor in nanoscale is of interest in the wide array of biomedical applications and environmental science for detection of metal ions and biomolecules possessing high sensitivity and low cost. The surface modified AuNCs can be utilized as biosensors with the fluorescent AuNCs core as transducer and the surface functionalized molecules as recognition component.

3.2.1. Heavy Metal Ions Detection. Due to high hazard and bioaccumulation in vital organs and tissues of heavy metal ions such as mercury, copper, and chromium ions, related investigations of heavy metal ions detection have received increasing attention. For the sake of protecting our environment and health, some effective strategies are highly desirable for the sensitive and selective detection of heavy metal ions.

The detection of $\mathrm{Hg}^{2+}$ is based on biomolecules (proteins $[129,130]$, antibodies [131], oligonucleotides [132], DNAzyme $[133,134]$, etc.), gold nanoparticles $[135,136]$, small organic molecules [137, 138], and other materials such as inorganic molecules [139, 140]. Recently, AuNCs have been exploited as the sensor of $\mathrm{Hg}^{2+}$. Xie et al. initially developed a method to synthesize AuNCs with BSA and further apply the BSA-templated AuNCs to the $\mathrm{Hg}^{2+}$ sensing in 2010 [141]. This approach utilized the specific and strong interaction between the $\mathrm{Au}^{+}$and $\mathrm{Hg}^{2+}$, which effectively quenched the fluorescence of AuNCs. This process can specifically detect and monitor $\mathrm{Hg}^{2+}$ ions at minimal concentration of $0.5 \mathrm{nM}$. Tseng and Lin have reported selectively sensing $\mathrm{Hg}^{2+}$ and $\mathrm{CH}_{3} \mathrm{Hg}^{+}$using lysozyme type VI-stabilized AuNCs (Lys VIAuNCs) through the interaction between $\mathrm{Hg}^{2+} / \mathrm{CH}_{3} \mathrm{Hg}^{+}$and $\mathrm{Au}^{+}$on the cluster surface, as shown in Figure 9. The limit of detection (LOD) for $\mathrm{Hg}^{2+} / \mathrm{CH}_{3} \mathrm{Hg}^{+}$was $3 \mathrm{pM}$ and $4 \mathrm{nM}$, respectively [54], which is much lower than the maximum concentration in the drinking water permitted by the U.S. Environmental Protection Agency (EPA).

Chang and colleagues have unveiled a new assay for the highly selective and sensitive detection of $\mathrm{Hg}^{2+}$ ions based on aggregation-induced quenching of the fluorescence of 11mercaptoundecanoic acid (11-MUA) protected AuNCs. This

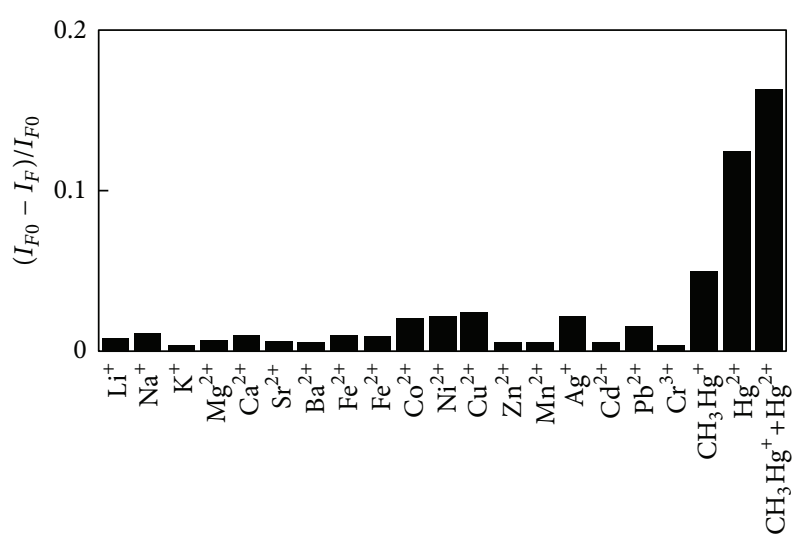

(a)

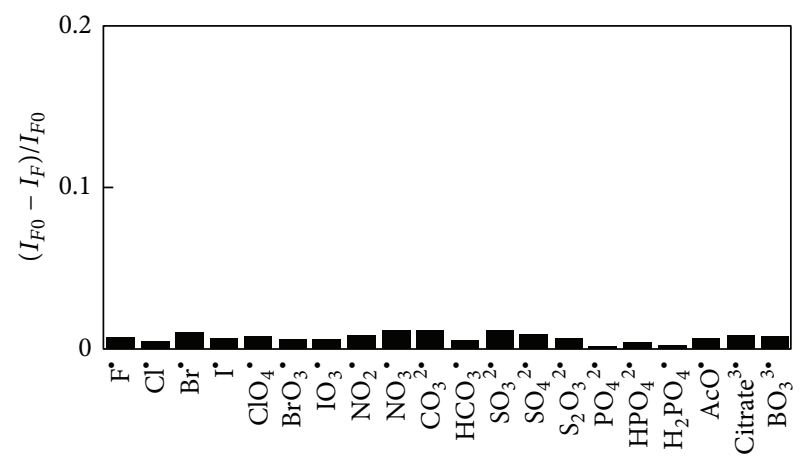

(b)

FIGURE 9: Relative fluorescence intensities $\left[\left(I_{F 0}-I_{F}\right) / I_{F 0}\right]$ at $631 \mathrm{~nm}$ of solutions of Lys VI-AuNCs after the addition of (a) $\mathrm{Hg}^{2+}$ $(100 \mathrm{nM}), \mathrm{CH}_{3} \mathrm{Hg}^{+}(100 \mathrm{nM})$, and other metal ions $(50 \mu \mathrm{M})$ and (b) anions $(50 \mu \mathrm{M})$. The excitation wavelength was set to $400 \mathrm{~nm}$. The incubation time was $10 \mathrm{~min}$. It should be noted that $I_{F 0}$ and $I_{F}$ are the fluorescence intensities of the AuNCs before and after adding analytes, respectively. Reprinted with permission from [54], (2010, Analytical Chemistry.

fluorescence quenching induced by the aggregation of gold NPs in the present of $\mathrm{Hg}^{2+}$ was firstly reported as a method for sensing metal ions [33].

Copper widely exists and is essential for all the plants and animals for being the key factor in producing numerous enzymes and involved in various physiologic processes; however, excessive copper ions will lead to cellular toxicity, liver damage, and other damages of organisms [54, 142, 143]. Thus, considerable efforts have been made in the field of $\mathrm{Cu}^{2+}$ detection using fluorescent AuNCs as a sensor. Gaetke and colleagues have demonstrated a novel method using GSHstabilized AuNCs as highly sensitive and selective fluorescent sensor for copper ions based on ion-induced aggregation of AuNCs [144]. In addition, the quenched fluorescence of AuNCs can be recovered through adding a strong metal ion chelator, ethylenediaminetetraacetate (EDTA). Guo and coworkers also reported a novel synthetic approach of NIR fluorescent AuNCs recently, which was realized by the heatassisted reduction of an $\mathrm{Au}^{+}$-thiol complex. The as-prepared AuNCs are able to selectively and sensitively detect copper 


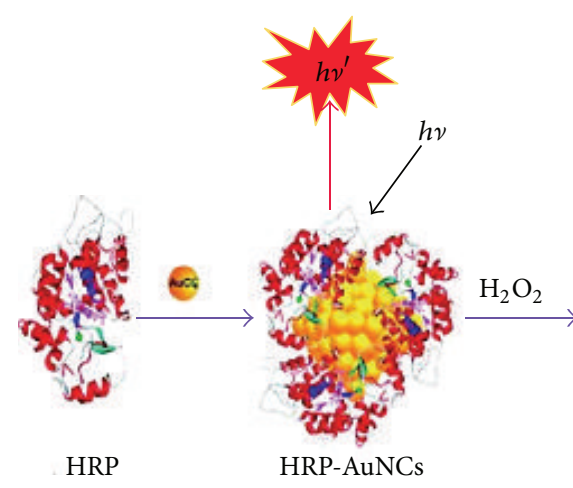

(a)
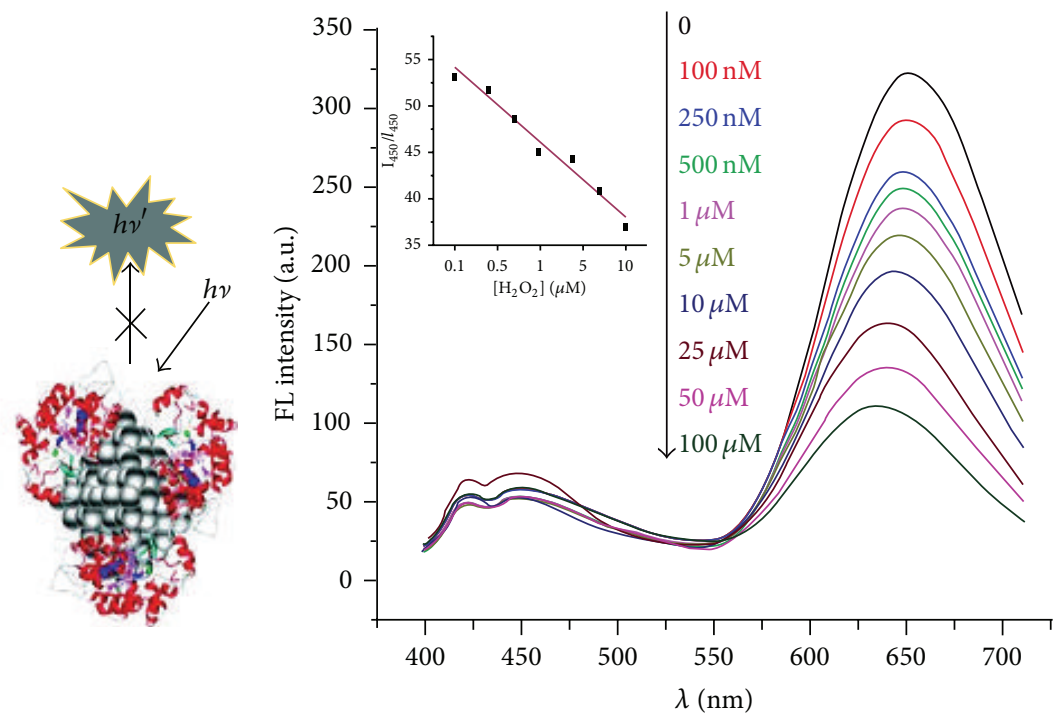

(b)

Figure 10: Schematic of the formation and the $\mathrm{H}_{2} \mathrm{O}_{2}$ directed quenching of HRP-AuNCs. Reprinted with permission from [127], @2011, American Chemical Society.

ions with a minimal concentration as low as $1.6 \mathrm{nM}[145]$. Guo and coworkers published the detection method of $\mathrm{Cu}^{2+}$ employing lysine-functionalized AuNCs (AuNCs@Lys) as fluorescent probes [146]. The mechanism of this sensing process is the tunable fluorescent signal of AuNCs@Lys quenched by the link of copper ions and lysine. The concentrate scale the probe can determine is from $10 \mathrm{nM}$ to $7 \mu \mathrm{M}$, which is far low than the maximum level of $\mathrm{Cu}$ in drinking water $(20 \mu \mathrm{M})$ permitted by the EPA of the U.S. [147]. A masking method for improving selectivity of AuNCs has been developed by Cao and coworkers for higher selective detection of mercury and copper ions. By adding the "masking" agents, respectively, $\mathrm{Cu}^{2+}$ and $\mathrm{Hg}^{2+}$ ions are inhibited to interact with AuNCs and eliminated the corresponding quenching effect; thus the ions without being inhibited can be detected [148].

Moreover, Ho and coworkers have described a facile onepot method to sense ferric ions by the fluorescent AuNCs stabilized and reduced by L-3, 4-dihydroxyphenylalanine (L-DOPA). The as-prepared AuNCs exhibited a fluorescent quench based on the ferric ions-induced aggregation, whose detection limit of the $\mathrm{Fe}^{3+}$ was lower than the maximum level allowed by the U.S. EPA $(5.4 \mu \mathrm{M})[149]$.

3.2.2. Small Molecules and Biomacromolecules Detection. In addition to heavy metal ion detection, fluorescent AuNCs can also be exploited detecting small molecules (hydrogen peroxide [40, 150], enzymes [151-153], dopamine [154-156], nitrite [157], cholesterol [158], folic acid [159], ciprofloxacin [160], etc.) and biological macromolecules (proteins [161166], glucose [40, 167], etc.). Zhang and coworkers have showed a biomineralization strategy for the detection of hydrogen peroxide by synthesizing the AuNCs by a functional template-Horseradish peroxidase (HRP) at physiological conditions [127]. The fluorescence of HRP-stabilized
AuNCs was quenched upon the addition of $\mathrm{H}_{2} \mathrm{O}_{2}$ in the range from $100 \mathrm{nM}$ to $100 \mu \mathrm{M}$ with a LOD of $30 \mathrm{nM}$, as shown in Figure 10.

The detection of biological thiols such as cysteine (Cys) and glutathione (GSH) has been extensively investigated. Cys plays an important role in biological system from the protein formation to being an antidote for alcoholic adverse reactions. GSH is the most abundant thiol in cells which participates in numerous biological reactions such as neutralizing free radicals and peroxides, HIV expression, and cancer therapy, which show that the altering level of GSH is related to the diabetes and HIV disease. So the level of GSH in biological organism is measured with great importance. Park and coworkers have elaborated the detection of biological thiols systematically by using the BSA-stabilized AuNCs. The method is based on $\mathrm{Hg}^{2+}$-induced fluorescence quenching through high affinity of $\mathrm{Au}^{+}$and $\mathrm{Hg}^{2+}$ and blocking this interaction by selective coordination of thiols with $\mathrm{Hg}^{2+}$. This fluorescence-based sensing method for biothiols has advantages such as simple steps, fast reaction time, and costeffectiveness. Recently, Huang et al. have reported a simple, immune-dependent, and label-free method for cystatin C (Cys C) detection. To achieve the detection, BSA-templated AuNCs have been employed as the fluorescent probe, which process is based on the papain-induced quenching of AuNCs and the fluorescent recovery by the coexistence of Cys C. Papain can digest the scaffold of AuNCs, BSA, based on its protease activity, and Cys $\mathrm{C}$ as a powerful cysteine proteinase inhibitors, capable of inhibiting the activity of papain to protect the AuNCs from aggregation. Fluorescent AuNCs enable detecting Cys $\mathrm{C}$ in the range of $25 \mathrm{ng} / \mathrm{mL}-$ $2.0 \mu \mathrm{g} / \mathrm{mL}$. Tseng et al. have also presented a method for sensing glutathione (GSH) by (Lysozyme Type VI) stabilized (Lys VI) AuNCs (contain 8 gold atoms). The PH-dependent 
blue-emitting AuNCs could detect GSH resulted from the formation of $\mathrm{GSH}-\mathrm{Au}^{+}$complexes. By specific etching reaction of GSH and AuNCs core, the fluorescence of AuNCs was quenched to show the altering level of GSH with a sensing limitation of $20 \mathrm{nM}$.

Dopamine (DA), as a vital catecholamine neurotransmitter, plays a significant role in the central nervous, renal, and hormonal systems [168]. The detection of DA with simple, high performance, nontoxicity has been developed employing the AuNCs as biosensor. $\mathrm{Qu}$ and coworkers have firstly discussed the detection of dopamine involving BSA-templated AuNCs electrogenerated chemiluminescence (ECL) study [155]. The process was realized by injecting DA into the electrolyte, the ECL of the AuNCs exhibited a distinct linear increase response to the increase of DA's concentration. The highly sensitive and selective fluorescence and colorimetric sensing of DA was in the first time described using a BSA-stabilized AuNCs by Sony and colleague [154]. The fluorescence of BSA-AuNCs showed a decrease once adding DA due to the photoinduced electron transfer of DA to AuNCs. In the meantime, the peroxidase-like activity of AuNCs was inhibited in the present of DA leading to a visual detection with highly selectivity.

AuNCs-based fluorescent protein sensor can be achieved by coupled selective recognition molecules with the fluorescent AuNCs. Of the substantial trial for forming AuNCsbased protein sensors, Leblanc's group initially reported the conjugation of anti-human IgG to the PAMAM-stabilized AuNCs for detecting the human IgG antigen [161]. Linear fluorescence quenching coordinated with the formation of antigen-antibody immunocomplexes. Chang and colleagues unveiled a novel protein sensor by using the light switching system based on biofunctional 11-MUA-protected AuNCs and bioconjugated $13 \mathrm{~nm}$ gold NPs [165]. Platelet-derived growth factor AA (PDGF AA), as a breast cancer marker protein, can be conjugated to AuNCs (PDGF AA- AA$\left.\mathrm{L}_{\mathrm{AuND}}\right)$. Thiol-derivation aptamers (Apt) with high affinity to PDGFs interacted with gold NPs (Apt-Q $\left.\mathrm{QuNP}_{\mathrm{AuN}}\right)$. PDGF AA$\mathrm{L}_{\mathrm{AuND}}$ can attach to the Apt- $\mathrm{Q}_{\text {AuNP }}$ leading to the fluorescence quenching by resonance energy transfer. Upon the addition of free PDGF or PDGF $\alpha$-receptor, the fluorescence can be recovered as a result of competitive reaction of PDGF/Apt$\mathrm{Q}_{\text {AuNP }}$ or PDGF $\alpha$-receptor/PDGF AA- $\mathrm{L}_{\mathrm{AuND}}$, as shown in Figure 11. The mannose-protected AuNCs have also been synthesized for the detection of Concanavalin A (Con A) with high sensitivity and other proteins and lectins [166]. In this method, the fluorescent AuNCs were synthesized by etching the THPC-reduced AuNCs by excessive Man$\mathrm{SH}$, which showed a high sensing limitation to Con $\mathrm{A}$ and E. coli attributed to multivalent cooperative interactions between Man-AuNCs and proteins. This approach provided the lowest LOD value for Con A (75 pM) and yielded brightly fluorescent cell clusters when binding to bacteria.

Glucose on one hand is the major energy source of living cells, of significance in the synthesis of other complex molecules, and an important factor of human health condition; on the other hand, it leads to many diseases such as diabetes when holding at an abnormal level. Typically, the detection of glucose by the fluorescent AuNCs probes is

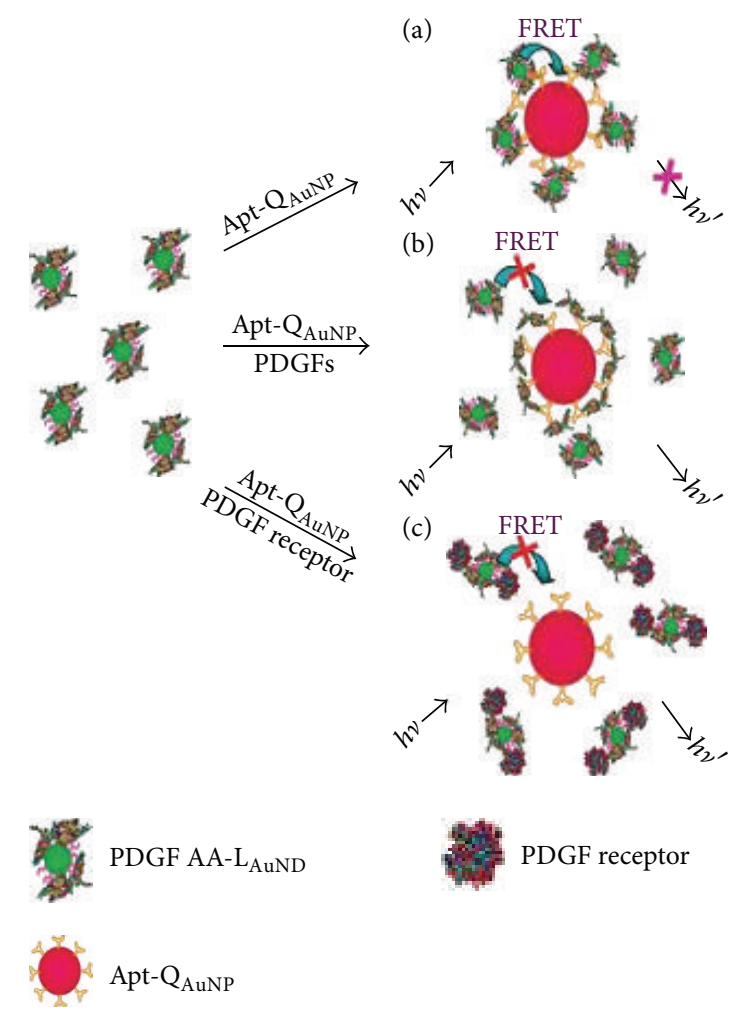

FIGURE 11: Schematic representations of PDGF and PDGF receptor biological nanosensors blocking the fluorescence quenching between PDGF AA- $\mathrm{L}_{\mathrm{AuND}}$ and Apt- $\mathrm{Q}_{\mathrm{AuNP}}$. Reprinted with permission from [165], ( $)(2008)$, American Chemical Society.

as follows: the biomolecules-stabilized AuNCs were synthesized; moreover, glucose can be oxidized by oxygen incubated with glucose oxidase (GOD) which produces $\mathrm{H}_{2} \mathrm{O}_{2}$ as the intermediation. The $\mathrm{H}_{2} \mathrm{O}_{2}$-induced aggregation of AuNCs can lead to the fluorescence quenching and vicariously detecting the glucose $[168,169]$.

3.3. Biological Labeling and Imaging. AuNCs, as an emerging fluorescent nanomaterial, possess a dramatic number of characteristics as follows: ultrasmall size, good water solubility, biocompatibility, photostability, and large Stokes shifts; all these properties make them promising fluorescent probes for biological labeling and imaging $[170,171]$.

The fluorescent AuNCs as cell labels are considered to realize the long observation times and stable emission of fluorescence microscopy in live cells so as to understand the dynamics of intracellular networks better. Parak et al. have prepared water-soluble fluorescent DHLA-protected AuNCs (AuNCs@DHLA), then bioconjugated with streptavidin for specific probing of the intracellular distribution of endogenous biotin in liver cell [16]. Thereafter, an investigation was also described by Chang and coworkers to elucidate the fluorescent and biocompatibility features of AuNCs by labeling the endothelial progenitor cells (EPC) [172]. The result indicated that the fluorescence of labeled cells did not decrease as well as maintained intact angiogenic potential. 


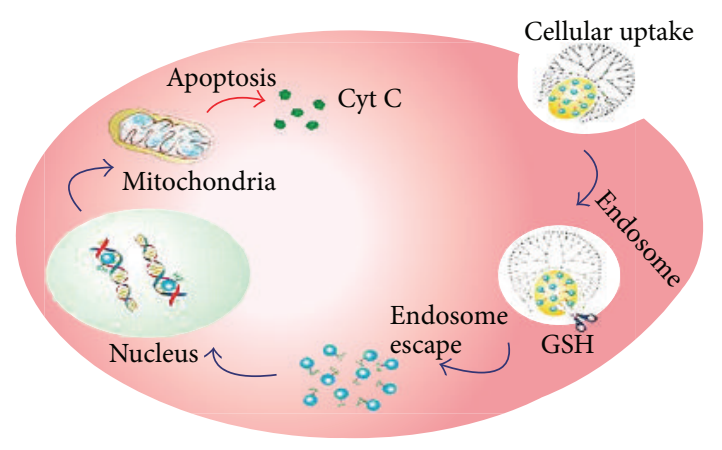

FIGURE 12: Intracellular GSH-triggered release of anticancer drugs loaded within DEGNPs. Reprinted with permission from [178], (C)2013, American Chemical Society.

Furthermore, AuNCs are attractive in the biomedicine field for diagnostics and therapeutics due to ultrasmall size, strong fluorescence emission and good biocompatibility [172-176]. Irudayaraj and coworkers have described the fluorescent BSA-protected AuNCs conjugated with Herceptin (AuNCs-Her) for targeting fluorescent imaging and cancer therapy at the same time [177]. The ultrafine novel fluorescent probes were demonstrated having the ability to enter the nucleus with high targeting specificity and nuclear localization capability for effective drug delivery and therapeutic efficacy. Botella and coworkers have designed a phototheranostic agent based on chlorin e6 (Ce6) photosensitizerconjugated silica-coated AuNCs (AuNC@SiO ${ }_{2}$-Ce6) for fluorescent imaging-guided photodynamic therapy [175]. And most recently, Cheng and colleagues have published the first report of using Dendrimer-encapsulated gold nanoparticles (DEGNPs) as the scaffold to develop stimuli-responsive drug delivery systems, as shown in Figure 12 [178]. The DEGNPs can be loaded with thiolates anticancer drugs via Au-S bond. After triggering to the tumor cell by GSH, the drugs can be released in tumors. This strategy opened up new possibilities to exploit subnanosized NPs for an "off-on" drug release system.

In addition to achieve more effective therapeutic effect, AuNCs-based probes can also be utilized for highly specific and accurate cancer diagnosis. Most recently, to achieve high accuracy and specificity of cancer diagnosis, novel fluorescence enzyme mimetic nanoprobes have been described by Chen et al. based on folic acid modified gold nanoclusters [173]. In this method, the BSA-stabilized AuNCs were synthesized to facilitate a rapid, cost-effective, and versatile NIR imaging/visualizing cancer diagnosis system. The peroxidase mimetic AuNCs conjugated to folic acid possess enhanced targeting ability to folate receptor over-expressing tumors, and combined with other superior properties, they can be employed for a colocalization staining method which diagnoses cancer rapidly through microscopic imaging with bright field and fluorescent imaging simultaneously.

Biological imaging in vivo is confronted with much more challenges than cellular imaging such as difficulty in survive in complex physiological environment and weak intensity of visible light blocking by organic tissue. The emitted light in the NIR region of AuNCs is capable of penetrating into deeper tissue and detecting stronger intensity due to weakest biological autofluorescence. Besides, unlike the toxic QDs and low-stability organic dyes, AuNCs with ultrafine size, good water-solubility, biocompatibility, and good photostability are of great interest to be fluorescent nanoprobes for biological imaging. As mentioned above, AuNCs are ideal bioprobes for bioimaging in vivo. Herein, we will show the latest development in bioimaging.

The two-photon excitation photoluminescence measurement was utilized to demonstrate the strong advantage in live cell imaging compared with organic dyes and quantum dots such as the ability of deeper inside tissues and the reduced phototoxicity of NIR light. Goodson and colleagues have investigated two-photo absorption (TPA) of $\mathrm{Au}_{25}$ clusters in NIR region in organic phase, and TPA cross-sections increase to an extremely high level at $800 \mathrm{~nm}$ which make it possible for applications in optical power limiting nanolithography [182]. Xu and coworkers have investigated the one- and two-photon excitation properties of water-soluble GSH-protected AuNCs and their applications in bioimaging [183]. In this report, the TPA property and application as fluorescence imaging contrast agents of GSH-AuNCs have been explored. The TPA cross section of GSH-AuNCs is much larger compared with organic dyes and QDs as well as the low toxicity and exceptional photostability which show their as promising in live cell fluorescent imaging and other bioimaging in vivo. Shang et al. have studied the process that DPA-AuNCs are internalized by HeLa cells in endosomal vesicles through two-photo imaging [41]. After 2 hours of incubation of DPA-AuNCs and HeLa cells, the images were captured by confocal microscopy with twophoton excitation. The fluorescence of probes ingested by the HeLa cells can easily be seen through 3D reconstruction images which reveal the nanoclusters inside the cells as well as those attached to the outside of the plasma membrane. The study of two-photon induced luminescence of 11-MUAAuNCs has been reported by Chou and co-workers. The two-photon induced fluorescent imaging was used to human mesenchymal stem cells (hMSCs) through the stable and nontoxicity nanoprobes, which revealed that the 11-MUAAuNCs were internalized into the cells and mainly resided in the cytoplasm nearby the nucleus, as shown in Figure 13 [179].

Compared with fluorescent intensity imaging, fluorescent lifetime imaging microscopy (FLIM) could separate the species based on differences in the exponential decay rate of fluorescence (lifetime), which is independent of the local concentration of fluorescent molecules and the excitation intensity [184]. Shang and colleagues have investigated the fluorescent DHLA-AuNCs in HeLa cells by the FLIM technique. The lifetimes of DHLA-AuNCs (>100 ns) is two order of magnitude longer than the lifetime of biological fluorescent fluorophores. Owing to this, the biological imaging can be achieved in the complete absence of cellular autofluorescence. The intensity and lifetime images of cell without and with AuNCs have been shown and indicated that the AuNCs have been internalized by the cells. The as-prepared NCs have many other features for promising candidate of wide applications in biomedical field. In the meantime, Irudayaraj 


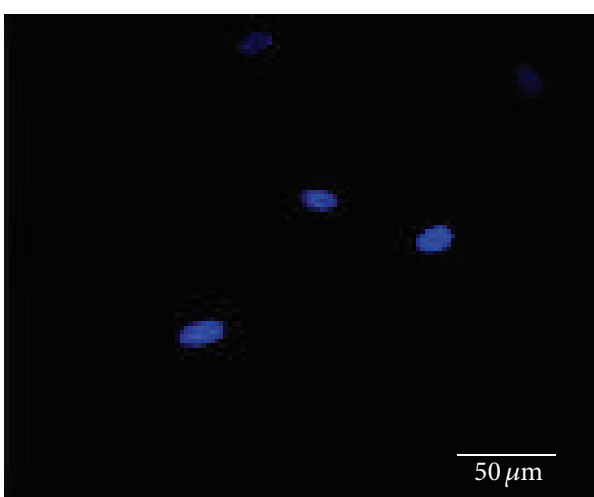

(a)

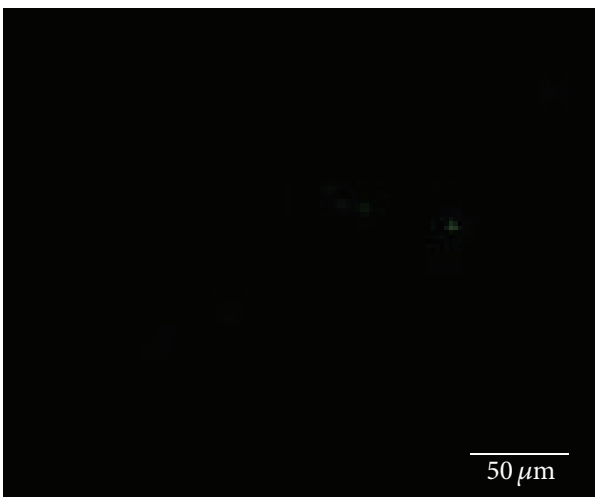

(c)

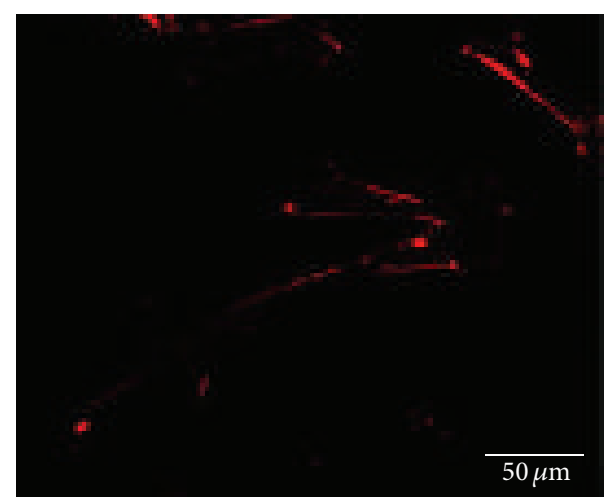

(b)

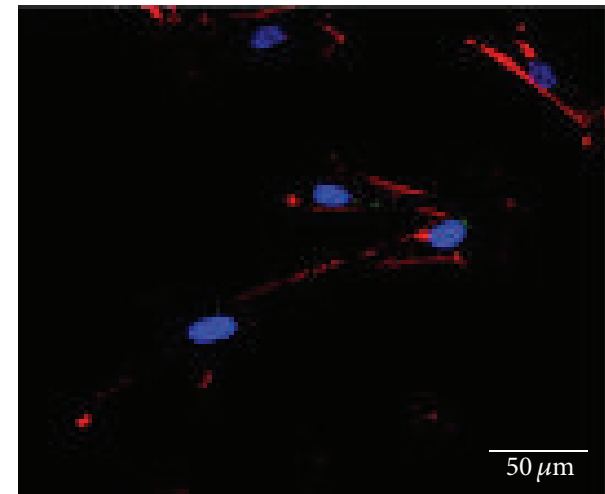

(d)

FIGURE 13: hMSCs were treated with dextran-coated 11MUA-Au nanodots for $24 \mathrm{~h}$ and then processed for two-photon and confocal microscopic examination. (a) Cell nucleus was stained with DAPI (blue color). (b) Actin fiber was stained with rhodamine phalloidin to confirm the cell boundary (red color). (c) Dextran-coated 11MUA-Au nanodots exhibit a two-photon emission (green luminescence) for in vitro bioapplication. (d) Fluorescence image overlay of the three images demonstrating the internalization of 11MUA-Au nanodots residing near the nucleus. Reprinted with permission from [179], (02009, American Chemical Society.

and workmates have exploited FLIM combined with fluorescent correlation spectroscopy (FCS) to track the diffusion of Herceptin-conjugated AuNCs and its entry into the nucleus in SK-BR3 cells [177]. The SK-BR3 cell is a human breast cancer cell overexpressing human epidermal growth factor receptor 2 (HER2) on the cell membrane. The AuNCs-Her was specifically endocytosed into the nucleus of SK-BR3 cells causing DNA damage showed by FLIM, as shown in Figure 14.

Biological imaging in vivo is confronted with much more challenges than cellular imaging such as difficulty in survive in complex physiological environment and weak intensity of visible light blocking by organic tissue. The emitted light in the NIR region of AuNCs is capable of penetrating into deeper tissue and detecting stronger intensity due to weakest biological autofluorescence. Besides, unlike the toxic QDs and low-stability organic dyes, AuNCs with ultrafine size, good water-solubility, biocompatibility, and good photostability are of great interest to be fluorescent nanoprobes for biological imaging. All of these properties indicates that AuNCs are ideal bioprobes for bioimaging in vivo. Herein, we will show the latest development in bioimaging.

Zhang et al. have developed the utilization of AuNCs into multimodal imaging technique for early cancer diagnosis
[185]. In this method, BSA-stabilized AuNCs are synthesized as the highly fluorescent and strong X-ray absorption coefficient contrast agent for fluorescent and X-ray dualmodality imaging. Coincidentally, Cui and coworkers have prepared FA-conjugated AuNCs@SiO 2 nanoprobes for in vivo dual-modal fluorescent and X-ray computed tomography gastric cancer targeting imaging [180]. Nude mice model with xenograft tumor based on MGC-803 cells was injected with these targeting fluorescent nanoprobes via tail vein followed by dual-modal imaging. The experimental results demonstrated that FA-conjugated AuNCs@SiO 2 can effectively target in vivo gastric cancer cells and exhibited excellent NIR fluorescent imaging and presented distinguished CT signals in X-ray imaging, as shown in Figure 15.

Moreover, a multimodal imaging method blending NIR fluorescent imaging and magnetic resonance imaging (MRI) has been achieved by Koyakutty and coworkers recently with the aid of gadolinium oxide and $\mathrm{Au}\left(\mathrm{Gd}_{2} \mathrm{O}_{3} / \mathrm{Au}\right)$ hybrid targeting nanoprobes [186]. The as-synthesized nanoclusters fabricated by biomineralization showed both intense redemitting fluorescence and good magnetic resonance imaging ability and were further functionalized with RGD peptide for realizing the targeted tumor imaging application in vivo. In addition, the over expression of $\alpha_{V} \beta_{3}$ integrin in U87 


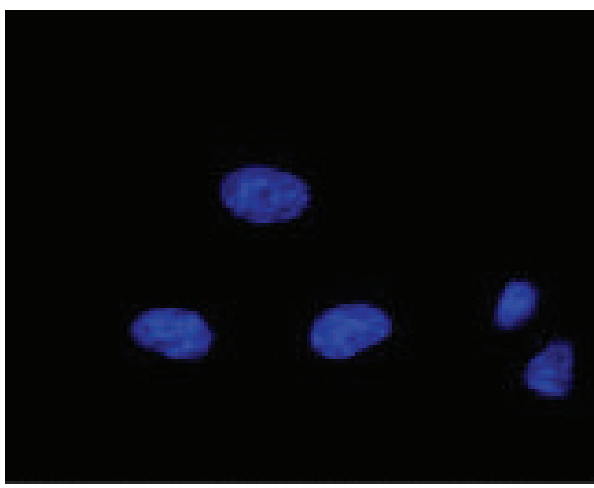

(a)

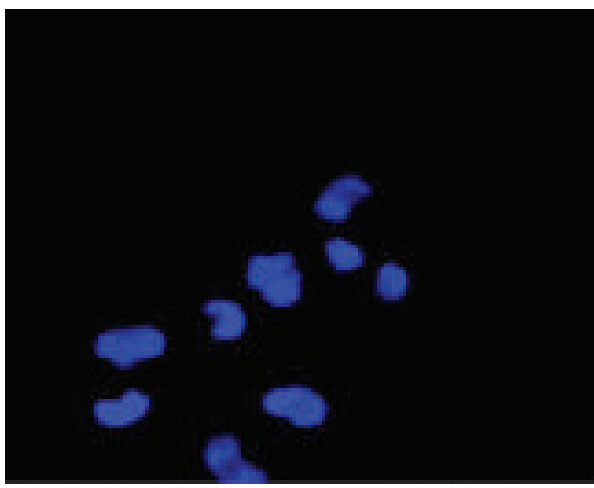

(b)

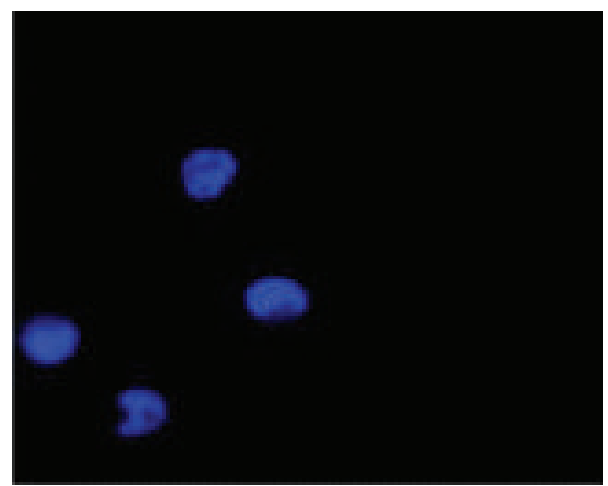

(c)

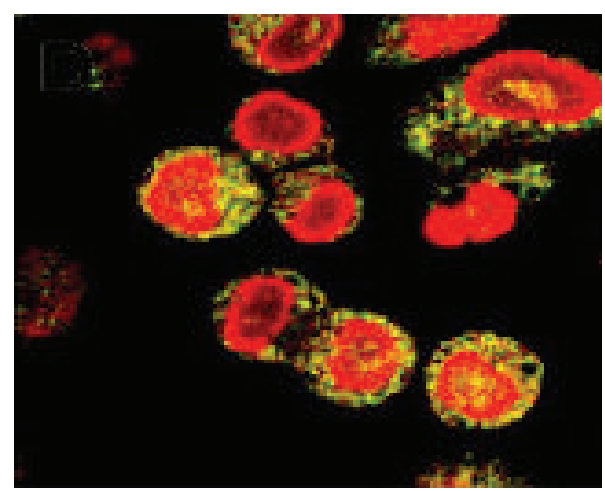

(d)

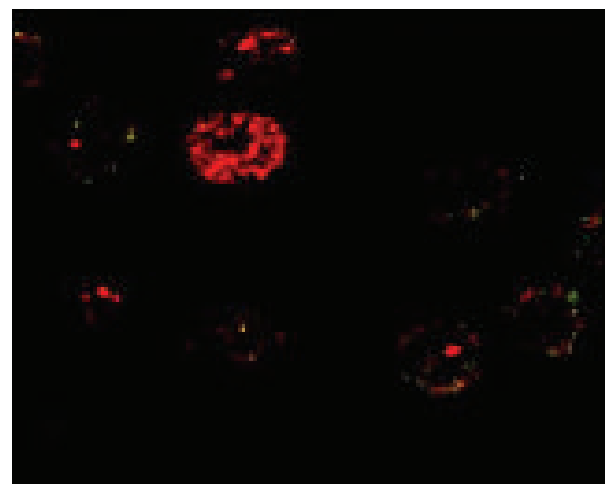

(e)

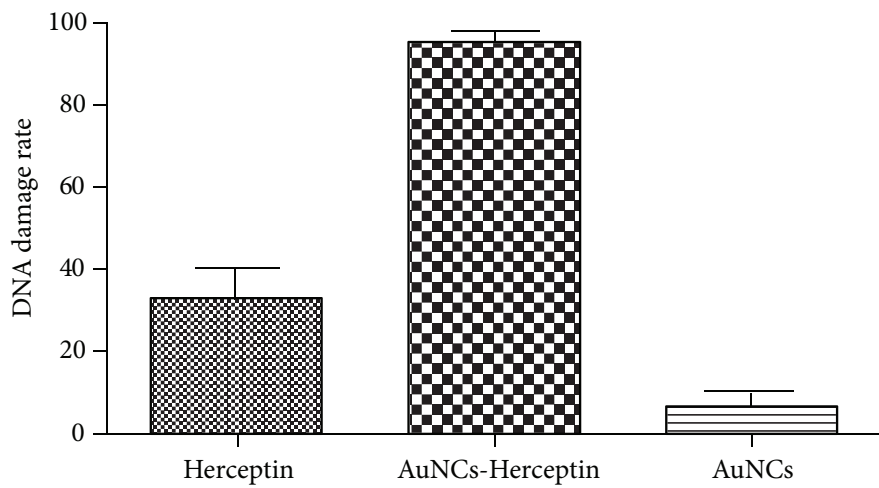

(f)

FIGURE 14: Fluorescent images showed the cell apoptosis induced by (a) AuNCs alone; (b) AuNCs-Her; (c) Herceptin by staining the nucleus with Hoechst 33258 (UV light $460 \mathrm{~nm}$ ). FLIM shows the DNA damage of SK-BR3 cells induced by (d) AuNCs-Her; (e) Herceptin indicated by the bright yellow dots. (f) Quantitative evaluation of DNA damage of cells as a percentage of the total number of cells for different treatments. Reprinted with permission from [177], (C2011, American Chemical Society.

tumor cell can enhance the specific accumulation of RGD$\mathrm{Gd}_{2} \mathrm{O}_{3} / \mathrm{Au}$. RGD conjugated $\mathrm{Gd}_{2} \mathrm{O}_{3} / \mathrm{Au}\left(\mathrm{RGD}-\mathrm{Gd}_{2} \mathrm{O}_{3} / \mathrm{Au}\right)$ nanoclusters injected into U87-MG tumor-bearing mice via tail vein showed distinguished specific accumulation in U87 tumor with longer retain time for targeted bioimaging in vivo. Currently, the majority of nanoprobes conjugated to targetable molecules are synthesized in labs followed by the purify steps and then injected into live animals. Although possessing ultrasmall size (nanoscale), relative stability in biological systems, and validated biocompatibility, the exogenous nanoprobes will reach the specific site through organic circulation, which inevitably triggers the clearance effect of biosystems. In order to overcome this problem, Wang and companions have created a biosynthesize method of AuNCs inside the cytoplasm through enforcing an efficient spontaneous reduction of chloroauric acid biocompatible salts by cancer cells, as shown in Figure 16 [181]. Although the mechanism of this novel bioimaging of cancer cells and tumors is still unknown, AuNCs have been successfully formed inside the cytoplasm and confirmed in vivo for specially labeling on the xenograft tumor mouse modal by fluorescent imaging. Thus, this novel method is remarkable not only for facilitating 


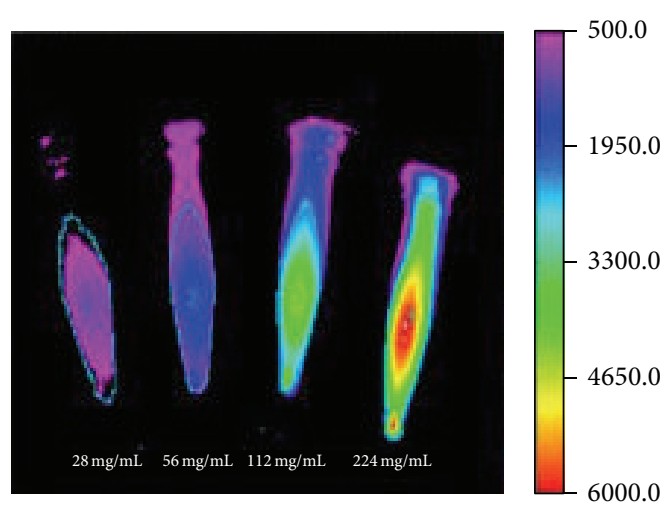

(a)

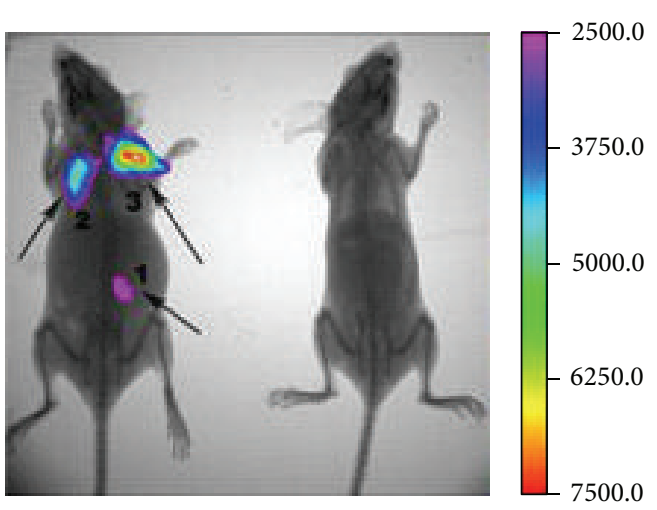

(b)

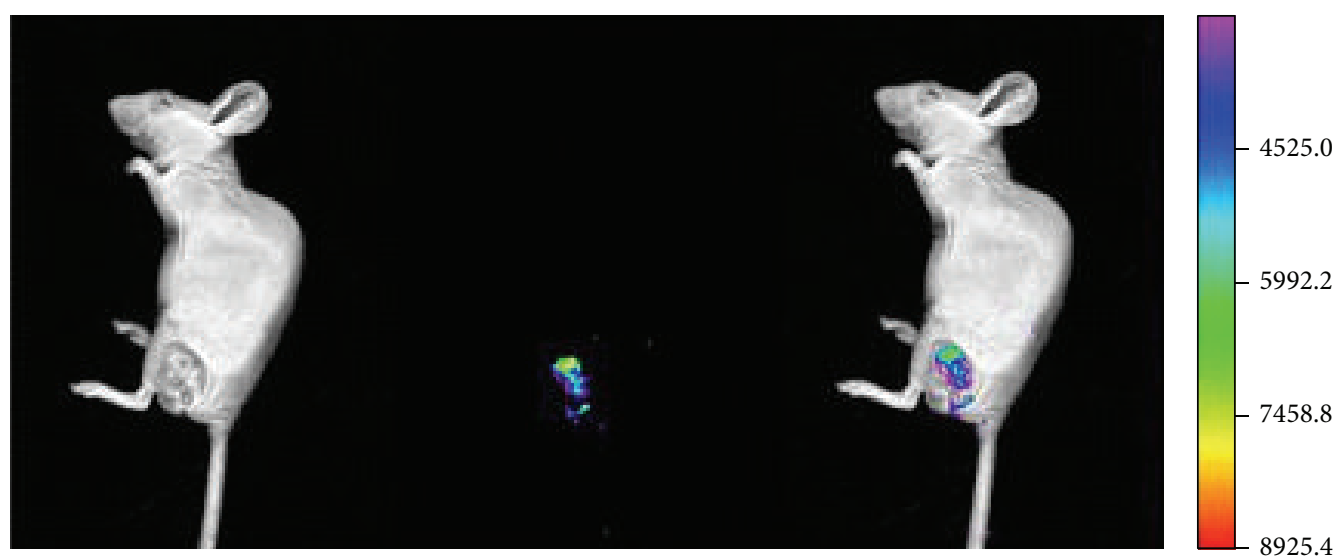

(c)

FIGURE 15: AuNCs@SiO 2 -FA nanoprobes for fluorescence imaging. (a) In vitro fluorescence image of AuNCs@SiO 2 -FA in 0.01 M PBS with different concentrations; (b) In vivo fluorescence image of $50 \mathrm{uL}$ AuNCs@SiO${ }_{2}$-FA injected subcutaneously at three different doses (area-1: $56 \mathrm{mg} / \mathrm{mL}$; area-2: $112 \mathrm{mg} / \mathrm{mL}$; area-3: $226 \mathrm{mg} / \mathrm{mL}$ ) into the left mice. The left mice without injection was selected as control; (c) fluorescence image of tumor tissues with tail vein injection at the concentration of $287 \mathrm{mg} / \mathrm{mL}$ at $6 \mathrm{~h}$ postinjection. Reprinted with permission from [180], (C)2013, Journal of Nanobiotechnology.

fluorescent nanoprobes with biosynthetic, self-imaging, and NPs circulation elusion peculiarities but also for showing a different concept of highly sensitive and specific nanoprobes for bioimaging application compared with current system.

\section{Summary and Outlook}

Fluorescent AuNCs, as an emerging fluorescent nanomaterial, possess a great deal of exceptional advantages such as good water-solubility, high photostability, large Stokes shift, ultrasmall size, nontoxicity, and good biocompatibility, compared with conventional organic dyes, rare earth-based quantum dots, and other luminescent materials. Herein, first of all, we have briefly summarized various approaches that have been employed for preparing AuNCs based on two routes: "bottom-up" and "top-down" routes. Recent advances of the development of the synthetic methods have a farreaching influence on the future functionalization of AuNCs. The surface modification of AuNCs endows a number of new functionalities for them like targeting imaging properties and therapeutic effects. In the second part, the most recent advances of bioapplications have been summed up such as fluorescent detection of metal ions/biomolecules, fluorescent cell labeling, and bioimaging in live animal.

Although a huge progression has been achieved to develop the promising novel fluorescent nanoprobes, a variety of challenges in the way still make the future progress of AuNCs.

With regard to the synthesis method and physical properties, there are several challenges we need to face in the future study. Even with making tremendous efforts to optimize the synthetic routes, problems remain existent such as the unpurified AuNCs being polydispersed in the solvent and exhibiting quantum yield lower than $20 \%$ within a broad emission band. What is more, there is scarce strategy for preparing size-focusing AuNCs with high reactive yield and monodisperse advantage. Finally, the relationship between the structure and optical properties of AuNCs requires deeper investigation.

For the application of AuNCs, most of biological applications focus on employing them as biosensors or fluorescent nanoprobes in bioimaging, and more applications involved in the therapy of tumor can be developed. Furthermore, the interaction between biological environment and AuNCs 

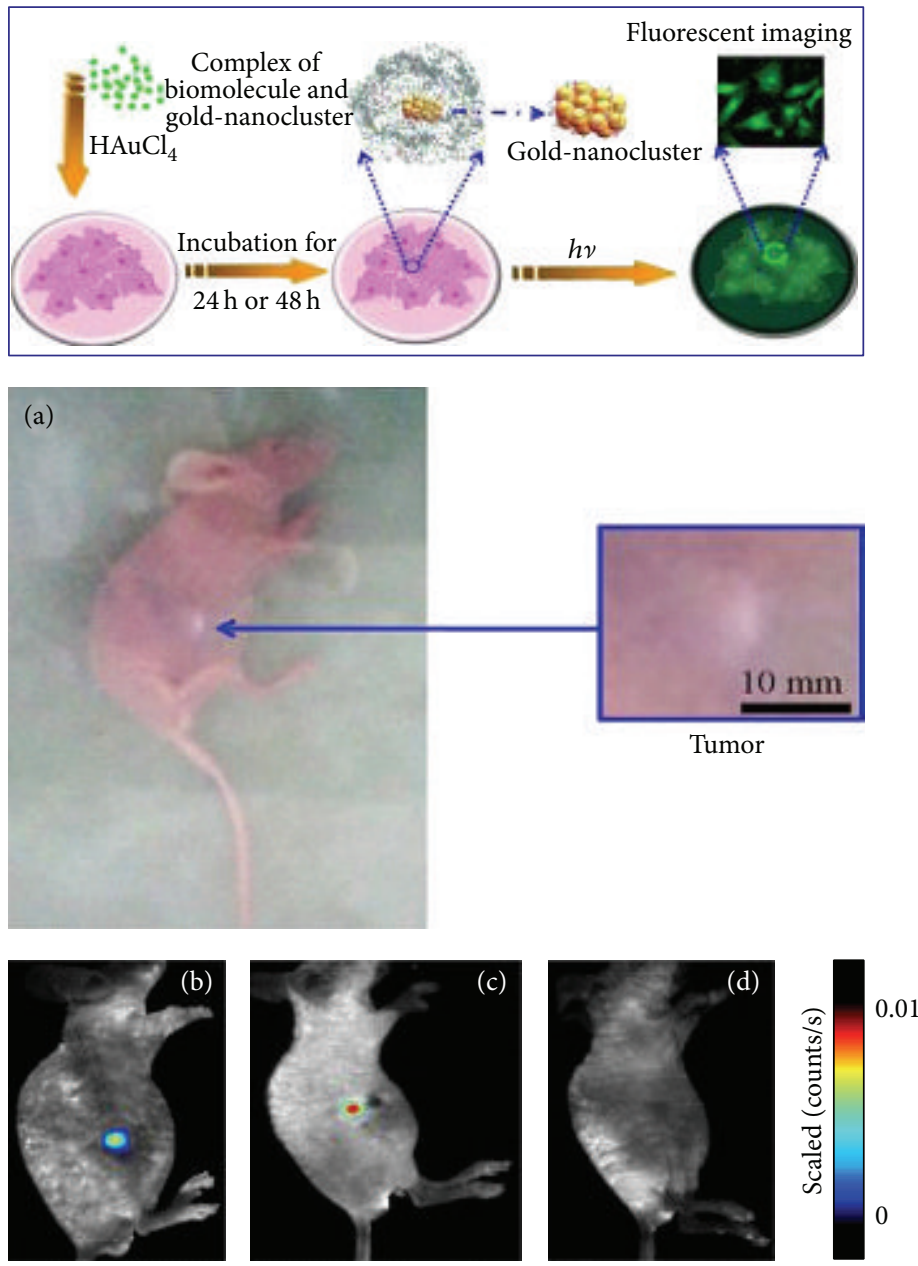

FIGURE 16: Schematic illustration of in situ biosynthesis of gold nanoclusters in cells and tumor imaging (top). Representative xenograft tumor mouse models of hepatocellular carcinoma observed in normal light (a) or by in vivo fluorescence imaging (b) $24 \mathrm{~h}$ after a subcutaneous injection of $10 \mathrm{mmol} / \mathrm{L} \mathrm{HAuCl}_{4}$ solution near the tumor; (c) $24 \mathrm{~h}$ after a subcutaneous injection of $10 \mathrm{mmol} / \mathrm{L} \mathrm{HAuCl}{ }_{4}$ solution near the tumor. (d) Control mouse observed by in vivo fluorescence imaging $48 \mathrm{~h}$ after a subcutaneous injection of $10 \mathrm{mmol} / \mathrm{L} \mathrm{HAuCl}_{4}$ solution in the right side of their abdomen (bottom). Reprinted from the permission of [181], Copyright @2013, Rights Managed by Nature Publishing Group.

is significant for the biological application of nanoprobes; however, most investigators judge the performance in organism centering in the matter of nanomaterial themselves (photostability, size-depending properties, etc.), but ignoring the interaction between the organism and AuNCs which is related to the safety of AuNCs used in the bionanotechnology and the mechanism of biological applications.

In a word, gold nanoclusters with fascinating properties have been extensively investigated and achieved marked advance. Nevertheless, the application of AuNCs in biological fields is still in the preliminary stage, and large amounts of study are waiting for us in the synthesis, comprehending of interaction mechanism, making full use of the decent features of AuNCs, and extending the range of biological applications.

\section{Conflict of Interests}

The authors declare that there is no conflict of interests regarding the publication of this paper.

\section{Acknowledgments}

This work is supported by the Program of the National Basic Research and Development Program of China (973) under Grant no. 2011CB707702, and the National Natural Science Foundation of China under Grant nos. 81371589, 81090272, and 81101084, and the Fundamental Research Funds for the Central Universities.

\section{References}

[1] E. C. Dreaden, A. M. Alkilany, X. Huang, C. J. Murphy, and M. A. El-Sayed, "The golden age: gold nanoparticles for biomedicine," Chemical Society Reviews, vol. 41, no. 7, pp. 27402779, 2012.

[2] M. C. Roco, "Nanotechnology: convergence with modern biology and medicine," Current Opinion in Biotechnology, vol. 14, no. 3, pp. 337-346, 2003. 
[3] L. Shang, S. Dong, and G. U. Nienhaus, "Ultra-small fluorescent metal nanoclusters: synthesis and biological applications," Nano Today, vol. 6, no. 4, pp. 401-418, 2011.

[4] M.-C. Daniel and D. Astruc, "Gold nanoparticles: assembly, supramolecular chemistry, quantum-size-related properties, and applications toward biology, catalysis, and nanotechnology," Chemical Reviews, vol. 104, no. 1, pp. 293-346, 2004.

[5] Z. Wu and R. Jin, "On the ligand's role in the fluorescence of gold nanoclusters," Nano Letters, vol. 10, no. 7, pp. 2568-2573, 2010.

[6] R. Jin, "Quantum sized, thiolate-protected gold nanoclusters," Nanoscale, vol. 2, no. 3, pp. 343-362, 2010.

[7] Q. Zhang, J. Xie, Y. Yu, and J. Y. Lee, "Monodispersity control in the synthesis of monometallic and bimetallic quasi-spherical gold and silver nanoparticles," Nanoscale, vol. 2, no. 10, pp. 19621975, 2010.

[8] L. Zhang and E. Wang, "Metal nanoclusters: new fluorescent probes for sensors and bioimaging," Nano Today, vol. 9, no. 1, pp. 132-157, 2014.

[9] J. Zheng, C. W. Zhang, and R. M. Dickson, "Highly fluorescent, water-soluble, size-tunable gold quantum dots," Physical Review Letters, vol. 93, no. 7, 2004.

[10] S. W. Chen, R. S. Ingram, M. J. Hostetler et al., "Gold nanoelectrodes of varied size: transition to molecule-like charging," Science, vol. 280, no. 5372, pp. 2098-2101, 1998.

[11] J. F. Hicks, A. C. Templeton, S. Chen et al., "The monolayer thickness dependence of quantized double-layer capacitances of monolayer-protected gold clusters," Analytical Chemistry, vol. 71, no. 17, pp. 3703-3711, 1999.

[12] J. F. Hicks, D. T. Miles, and R. W. Murray, "Quantized doublelayer charging of highly monodisperse metal nanoparticles," Journal of the American Chemical Society, vol. 124, no. 44, pp. 13322-13328, 2002.

[13] B. M. Quinn, P. Liljeroth, V. Ruiz, T. Laaksonen, and K. Kontturi, "Electrochemical resolution of 15 oxidation states for monolayer protected gold nanoparticles," Journal of the American Chemical Society, vol. 125, no. 22, pp. 6644-6645, 2003.

[14] K. S. Park, M. I. Kim, M.-A. Woo, and H. G. Park, "A labelfree method for detecting biological thiols based on blocking of $\mathrm{Hg}^{2+}$-quenching of fluorescent gold nanoclusters," Biosensors \& Bioelectronics, vol. 45, no. 1, pp. 65-69, 2013.

[15] N. L. Rosi and C. A. Mirkin, "Nanostructures in biodiagnostics," Chemical Reviews, vol. 105, no. 4, pp. 1547-1562, 2005.

[16] C.-A. J. Lin, T.-Y. Yang, C.-H. Lee et al., "Synthesis, characterization, and bioconjugation of fluorescent gold nanoclusters toward biological labeling applications," ACS Nano, vol. 3, no. 2, pp. 395-401, 2009.

[17] F. Wang, W. B. Tan, Y. Zhang, X. P. Fan, and M. Q. Wang, "Luminescent nanomaterials for biological labelling," Nanotechnology, vol. 17, no. 1, pp. R1-R13, 2006.

[18] G. Wang, T. Huang, R. W. Murray, L. Menard, and R. G. Nuzzo, "Near-IR luminescence of monolayer-protected metal clusters," Journal of the American Chemical Society, vol. 127, no. 3, pp. 812813, 2005.

[19] X. Li, F. Zhang, and D. Zhao, "Highly efficient lanthanide upconverting nanomaterials: progresses and challenges," Nano Today, vol. 8, no. 6, pp. 643-676, 2013.

[20] I. Díez and R. H. A. Ras, "Fluorescent silver nanoclusters," Nanoscale, vol. 3, no. 5, pp. 1963-1970, 2011.

[21] H. Xu and K. S. Suslick, "Water-soluble fluorescent silver nanoclusters," Advanced Materials, vol. 22, no. 10, pp. 1078-1082, 2010.
[22] M. A. H. Muhammed and T. Pradeep, "Luminescent quantum clusters of gold as bio-labels," in Advanced Fluorescence Reporters in Chemistry and Biology II, vol. 9 of Springer Series on Fluorescence, pp. 333-353, Springer, Berlin, Germany, 2010.

[23] I. Díez and R. H. A. Ras, "Few-atom silver clusters as fluorescent reporters," in Advanced Fluorescence Reporters in Chemistry and Biology II, vol. 9 of Springer Series on Fluorescence, pp. 307-332, Springer, Berlin, Germany, 2010.

[24] C.-A. J. Lin, C.-H. Lee, J.-T. Hsieh et al., "Synthesis of fluorescent metallic nanoclusters toward biomedical application: recent progress and present challenges," Journal of Medical and Biological Engineering, vol. 29, no. 6, pp. 276-283, 2009.

[25] J. Zheng, Fluorescent Noble Metal Nanoclusters, Georgia Institute of Technology, 2005.

[26] J. Zheng, P. R. Nicovich, and R. M. Dickson, "Highly fluorescent noble-metal quantum dots," Annual Review of Physical Chemistry, vol. 58, pp. 409-431, 2007.

[27] G. K. Wertheim, S. B. DiCenzo, and D. N. E. Buchanan, "Noble- and transition-metal clusters: the $\mathrm{d}$ bands of silver and palladium," Physical Review B, vol. 33, no. 8, pp. 5384-5390, 1986.

[28] S. Wang, X. Meng, A. Das et al., "A 200-fold quantum yield boost in the photoluminescence of silver-doped $\mathrm{Ag}_{x} \mathrm{Au}_{25-x}$ nanoclusters: the 13 th silver atom matters," Angewandte ChemieInternational Edition, vol. 53, no. 9, pp. 2376-2380, 2014.

[29] G. A. Ozin and S. A. Mitchell, "Ligand-free metal clusters," Angewandte Chemie International Edition, vol. 22, no. 9, pp. 674-694, 1983.

[30] Z. Q. Zhang, R. C. Patel, R. Kothari, C. P. Johnson, S. E. Friberg, and P. A. Aikens, "Stable silver clusters and nanoparticles prepared in polyacrylate and inverse micellar solutions," Journal of Physical Chemistry B, vol. 104, no. 6, pp. 1176-1182, 2000.

[31] T. Huang and R. W. Murray, "Visible luminescence of watersoluble monolayer-protected gold clusters," Journal of Physical Chemistry B, vol. 105, no. 50, pp. 12498-12502, 2001.

[32] S. Link, A. Beeby, S. FitzGerald, M. A. El-Sayed, T. G. Schaaff, and R. L. Whetten, "Visible to infrared luminescence from a 28atom gold cluster," The Journal of Physical Chemistry B, vol. 106, no. 13, pp. 3410-3415, 2002.

[33] C.-C. Huang, Z. Yang, K.-H. Lee, and H.-T. Chang, "Synthesis of highly fluorescent gold nanoparticles for sensing mercury(II)," Angewandte Chemie International Edition, vol. 46, no. 36, pp. 6824-6828, 2007.

[34] J. Zheng, J. T. Petty, and R. M. Dickson, "High quantum yield blue emission from water-soluble $\mathrm{Au}_{8}$ nanodots," Journal of the American Chemical Society, vol. 125, no. 26, pp. 7780-7781, 2003.

[35] N. Schaeffer, B. Tan, C. Dickinson et al., "Fluorescent or not? Size-dependent fluorescence switching for polymer-stabilized gold clusters in the 1.1-1.7 nm size range," Chemical Communications, no. 34, pp. 3986-3988, 2008.

[36] B. Santiago González, M. J. Rodríguez, C. Blanco, J. Rivas, M. A. López-Quintela, and J. M. G. Martinho, "One step synthesis of the smallest photoluminescent and paramagnetic PVP-protected gold atomic clusters," Nano Letters, vol. 10, no. 10, pp. 4217-4221, 2010.

[37] J. Xie, Y. Zheng, and J. Y. Ying, "Protein-directed synthesis of highly fluorescent gold nanoclusters," Journal of the American Chemical Society, vol. 131, no. 3, pp. 888-889, 2009.

[38] Y. Kong, J. Chen, F. Gao et al., "Near-infrared fluorescent ribonuclease-A-encapsulated gold nanoclusters: preparation, characterization, cancer targeting and imaging," Nanoscale, vol. 5, no. 3, pp. 1009-1017, 2013. 
[39] W.-Y. Chen, G.-Y. Lan, and H.-T. Chang, "Use of fluorescent DNA-templated gold/silver nanoclusters for the detection of sulfide ions," Analytical Chemistry, vol. 83, no. 24, pp. 94509455, 2011.

[40] J. A. Annie Ho, H. C. Chang, and W. T. Su, "DOPA-mediated reduction allows the facile synthesis of fluorescent gold nanoclusters for use as sensing probes for ferric ions," Analytical Chemistry, vol. 84, no. 7, pp. 3246-3253, 2012.

[41] L. Shang, R. M. Dörlich, S. Brandholt et al., "Facile preparation of water-soluble fluorescent gold nanoclusters for cellular imaging applications," Nanoscale, vol. 3, no. 5, pp. 2009-2014, 2011.

[42] V. Venkatesh, A. Shukla, S. Sivakumar, and S. Verma, "Purinestabilized green fluorescent gold nanoclusters for cell nuclei imaging applications," ACS Applied Materials and Interfaces, vol. 6, no. 3, pp. 2185-2191, 2014.

[43] X. Liu, C. Li, J. Xu et al., "Surfactant-free synthesis and functionalization of highly fluorescent gold quantum dots," Journal of Physical Chemistry C, vol. 112, no. 29, pp. 10778-10783, 2008.

[44] C. Zhou, C. Sun, M. Yu et al., "Luminescent gold nanoparticles with mixed valence states generated from dissociation of polymeric Au(I) thiolates," Journal of Physical Chemistry C, vol. 114, no. 17, pp. 7727-7732, 2010.

[45] Z. Luo, X. Yuan, Y. Yu et al., "From aggregation-induced emission of $\mathrm{Au}(\mathrm{I})$-thiolate complexes to ultrabright $\mathrm{Au}(0) @ \mathrm{Au}(\mathrm{I})$ thiolate core-shell nanoclusters," Journal of the American Chemical Society, vol. 134, no. 40, pp. 16662-16670, 2012.

[46] Y. Guo, Z. Wang, H. Shao, and X. Jiang, "Stable fluorescent gold nanoparticles for detection of $\mathrm{Cu}^{2+}$ with good sensitivity and selectivity," Analyst, vol. 137, no. 2, pp. 301-304, 2012.

[47] F. Aldeek, M. A. H. Muhammed, G. Palui, N. Zhan, and H. Mattoussi, "Growth of highly fluorescent polyethylene glycoland zwitterion-functionalized gold nanoclusters," ACS Nano, vol. 7, no. 3, pp. 2509-2521, 2013.

[48] T. U. B. Rao and T. Pradeep, "Luminescent $\mathrm{Ag}_{7}$ and $\mathrm{Ag}_{8}$ clusters by interfacial synthesis," Angewandte Chemie-International Edition, vol. 49, no. 23, pp. 3925-3929, 2010.

[49] J.-I. Nishigaki, S. Yamazoe, S. Kohara et al., "A twisted biicosahedral Au25 cluster enclosed by bulky arenethiolates," Chemical Communications, vol. 50, no. 7, pp. 839-841, 2014.

[50] V. R. Jupally and A. Dass, "Synthesis of $\mathrm{Au}_{130}(\mathrm{SR})_{50}$ and $\mathrm{Au}_{130-x} \mathrm{Ag}_{x}(\mathrm{SR})_{50}$ nanomolecules through core size conversion of larger metal clusters," Physical Chemistry Chemical Physics, vol. 16, no. 22, pp. 10473-10479, 2014.

[51] X. Yuan, Z. Luo, Q. Zhang et al., "Synthesis of highly fluorescent metal (Ag, $\mathrm{Au}, \mathrm{Pt}$, and $\mathrm{Cu}$ ) nanoclusters by electrostatically induced reversible phase transfer," ACS Nano, vol. 5, no. 11, pp. 8800-8808, 2011.

[52] X. Huang, Y. Luo, Z. Li et al., "Biolabeling hematopoietic system cells using near-infrared fluorescent gold nanoclusters," The Journal of Physical Chemistry C, vol. 115, no. 34, pp. 16753-16763, 2011.

[53] D. Joseph and K. E. Geckeler, "Synthesis of highly fluorescent gold nanoclusters using egg white proteins," Colloids and Surfaces B: Biointerfaces, vol. 115, pp. 46-50, 2014.

[54] Y.-H. Lin and W.-L. Tseng, "Ultrasensitive sensing of $\mathrm{Hg}_{2}{ }^{+}$and $\mathrm{CH}_{3} \mathrm{Hg}^{+}$based on the fluorescence quenching of lysozyme Type VI-stabilized gold nanoclusters," Analytical Chemistry, vol. 82, no. 22, pp. 9194-9200, 2010.

[55] J. Zheng and R. M. Dickson, "Individual water-soluble dendrimer-encapsulated silver nanodot fluorescence," Journal of the American Chemical Society, vol. 124, no. 47, pp. 1398213983, 2002.

[56] C. I. Richards, S. Choi, J.-C. Hsiang et al., "Oligonucleotidestabilized Ag nanocluster fluorophores," Journal of the American Chemical Society, vol. 130, no. 15, pp. 5038-5039, 2008.

[57] B. Han and E. Wang, "DNA-templated fluorescent silver nanoclusters," Analytical and Bioanalytical Chemistry, vol. 402, no. 1, pp. 129-138, 2012.

[58] D. A. Tomalia and J. M. J. Fréchet, "Discovery of dendrimers and dendritic polymers: a brief historical perspective," Journal of Polymer Science, Part A: Polymer Chemistry, vol. 40, no. 16, pp. 2719-2728, 2002.

[59] D. A. Tomalia, "Birth of a new macromolecular architecture: dendrimers as quantized building blocks for nanoscale synthetic organic chemistry," Aldrichimica Acta, vol. 37, no. 2, pp. $39-57,2004$.

[60] B. Yuping, Z. Chang, D. M. Vu, J. P. Temirov, R. B. Dyer, and J. S. Martinez, "Nanoparticle-free synthesis of fluorescent gold nanoclusters at physiological temperature," The Journal of Physical Chemistry C, vol. 111, no. 33, pp. 12194-12198, 2007.

[61] Y.-C. Jao, M.-K. Chen, and S.-Y. Lin, "Enhanced quantum yield of dendrimer-entrapped gold nanodots by a specific ionpair association and microwave irradiation for bioimaging," Chemical Communications, vol. 46, no. 15, pp. 2626-2628, 2010.

[62] L. Maya, G. Muralidharan, T. G. Thundat, and E. A. Kenik, "Polymer-mediated assembly of gold nanoclusters," Langmuir, vol. 16, no. 24, pp. 9151-9154, 2000.

[63] X. Huang, B. Li, L. Li et al., "Facile preparation of highly blue fluorescent metal nanoclusters in organic media," Journal of Physical Chemistry C, vol. 116, no. 1, pp. 448-455, 2012.

[64] H. Duan and S. Nie, "Etching colloidal gold nanocrystals with hyperbranched and multivalent polymers: a new route to fluorescent and water-soluble atomic clusters," Journal of the American Chemical Society, vol. 129, no. 9, pp. 2412-2413, 2007.

[65] I. Hussain, S. Graham, Z. X. Wang et al., "Size-controlled synthesis of near-monodisperse gold nanoparticles in the 1$4 \mathrm{~nm}$ range using polymeric stabilizers," Journal of the American Chemical Society, vol. 127, no. 47, pp. 16398-16399, 2005.

[66] Z. Wang, B. Tan, I. Hussain et al., "Design of polymeric stabilizers for size-controlled synthesis of monodisperse gold nanoparticles in water," Langmuir, vol. 23, no. 2, pp. 885-895, 2007.

[67] Y. Chen, X. Zheng, X. Wang, C. Wang, Y. Ding, and X. Jiang, "Near-infrared emitting gold cluster-poly(acrylic acid) hybrid nanogels," ACS Macro Letters, vol. 3, no. 1, pp. 74-76, 2014.

[68] C.-L. Liu, H.-T. Wu, Y.-H. Hsiao et al., "Insulin-directed synthesis of fluorescent gold nanoclusters: preservation of insulin bioactivity and versatility in cell imaging," Angewandte Chemie International Edition, vol. 50, no. 31, pp. 7056-7060, 2011.

[69] A. R. Garcia, I. Rahn, S. Johnson et al., "Human insulin fibrilassisted synthesis of fluorescent gold nanoclusters in alkaline media under physiological temperature," Colloids and Surfaces B: Biointerfaces, vol. 105, pp. 167-172, 2013.

[70] C. Guo and J. Irudayaraj, "Fluorescent Ag clusters via a proteindirected approach as a $\mathrm{Hg}(\mathrm{II})$ ion sensor," Analytical Chemistry, vol. 83, no. 8, pp. 2883-2889, 2011.

[71] H. Wei, Z. Wang, L. Yang, S. Tian, C. Hou, and Y. Lu, "Lysozyme-stabilized gold fluorescent cluster: synthesis and application as $\mathrm{Hg}^{2+}$ sensor," Analyst, vol. 135, no. 6, pp. 14061410, 2010. 
[72] J. Qiao, X. Mu, L. Qi, J. Deng, and L. Mao, "Folic acidfunctionalized fluorescent gold nanoclusters with polymers as linkers for cancer cell imaging," Chemical Communications, vol. 49, no. 73, pp. 8030-8032, 2013.

[73] E. S. Kryachko and F. Remade, "Complexes of DNA bases and Watson-Crick base pairs with small neutral gold clusters," Journal of Physical Chemistry B, vol. 109, no. 48, pp. 2274622757, 2005.

[74] M. K. Shukla, M. Dubey, E. Zakar, and J. Leszczynski, "DFT investigation of the interaction of gold nanoclusters with nucleic acid base guanine and the watson-crick guanine-cytosine base pair," Journal of Physical Chemistry C, vol. 113, no. 10, pp. 39603966, 2009.

[75] R. Zhou, M. Shi, X. Chen, M. Wang, and H. Chen, "Atomically monodispersed and fluorescent sub-nanometer gold clusters created by biomolecule-assisted etching of nanometer-sized gold particles and rods," Chemistry - A European Journal, vol. 15, no. 19, pp. 4944-4951, 2009.

[76] T. A. C. Kennedy, J. L. MacLean, and J. Liu, "Blue emitting gold nanoclusters templated by poly-cytosine DNA at low $\mathrm{pH}$ and poly-adenine DNA at neutral pH," Chemical Communications, vol. 48, no. 54, pp. 6845-6847, 2012.

[77] G. Liu, Y. Shao, K. Ma, Q. Cui, F. Wu, and S. Xu, "Synthesis of DNA-templated fluorescent gold nanoclusters," Gold Bulletin, vol. 45, no. 2, pp. 69-74, 2012.

[78] H. Kawasaki, H. Yamamoto, H. Fujimori, R. Arakawa, Y. Iwasaki, and M. Inada, "Stability of the DMF-protected Au nanoclusters: photochemical, dispersion, and thermal properties," Langmuir, vol. 26, no. 8, pp. 5926-5933, 2010.

[79] S. Palmal, S. Basiruddin, A. R. Maity, S. C. Ray, and N. R. Jana, "Thiol-directed synthesis of highly fluorescent gold clusters and their conversion into stable imaging nanoprobes," Chemistry, vol. 19, no. 3, pp. 943-949, 2013.

[80] H. Chen, B. Li, C. Wang et al., "Characterization of a fluorescence probe based on gold nanoclusters for cell and animal imaging," Nanotechnology, vol. 24, no. 5, Article ID 055704, 2013.

[81] Y. Zhang, Q. Hu, M. C. Paau et al., "Probing histidine-stabilized gold nanoclusters product by high-performance liquid chromatography and mass spectrometry," Journal of Physical Chemistry C, vol. 117, no. 36, pp. 18697-18708, 2013.

[82] X. Mu, L. Qi, P. Dong et al., "Facile one-pot synthesis of 1proline-stabilized fluorescent gold nanoclusters and its application as sensing probes for serum iron," Biosensors and Bioelectronics, vol. 49, pp. 249-255, 2013.

[83] X. Yang, M. Shi, R. Zhou, X. Chen, and H. Chen, "Blending of $\mathrm{HAuCl}_{4}$ and histidine in aqueous solution: a simple approach to the $\mathrm{Au}_{10}$ cluster.," Nanoscale, vol. 3, no. 6, pp. 2596-2601, 2011.

[84] R. de la Rica, L. W. Chow, C.-M. Horejs et al., "A designer peptide as a template for growing Au nanoclusters," Chemical Communications, vol. 50, no. 73, pp. 10648-10650, 2014.

[85] Y. Wang, Y. Cui, Y. Zhao et al., "Bifunctional peptides that precisely biomineralize Au clusters and specifically stain cell nuclei," Chemical Communications, vol. 48, no. 6, pp. 871-873, 2012.

[86] Y. Bao, H.-C. Yeh, C. Zhong et al., "Formation and stabilization of fluorescent gold nanoclusters using small molecules," Journal of Physical Chemistry C, vol. 114, no. 38, pp. 15879-15882, 2010.

[87] C. A. Simpson, C. L. Farrow, P. Tian et al., "Tiopronin gold nanoparticle precursor forms aurophilic ring tetramer," Inorganic Chemistry, vol. 49, no. 23, pp. 10858-10866, 2010.
[88] X. Yuan, Y. Yu, Q. Yao, Q. Zhang, and J. Xie, "Fast synthesis of thiolated $\mathrm{Au}_{25}$ nanoclusters via protection-deprotection method," Journal of Physical Chemistry Letters, vol. 3, no. 17, pp. 2310-2314, 2012.

[89] Y. Bao, C. Zhong, D. M. Vu, J. P. Temirov, R. B. Dyer, and J. S. Martinez, "Nanoparticle-free synthesis of fluorescent gold nanoclusters at physiological temperature," The Journal of Physical Chemistry C, vol. 111, no. 33, pp. 12194-12198, 2007.

[90] Y. Shichibu, Y. Negishi, T. Tsukuda, and T. Teranishi, "Largescale synthesis of thiolated $\mathrm{Au}_{25}$ clusters via ligand exchange reactions of phosphine-stabilized $\mathrm{Au}_{11}$ clusters," Journal of the American Chemical Society, vol. 127, no. 39, pp. 13464-13465, 2005.

[91] M. Yu, C. Zhou, J. Liu, J. D. Hankins, and J. Zheng, "Luminescent gold nanoparticles with $\mathrm{pH}$-dependent membrane adsorption," Journal of the American Chemical Society, vol. 133, no. 29, pp. 11014-11017, 2011.

[92] M. McPartlin, R. Mason, and L. Malatesta, "Novel cluster complexes of gold(0)-gold(I)," Journal of the Chemical Society D: Chemical Communications, no. 7, p. 334, 1969.

[93] B. K. Teo, X. Shi, and H. Zhang, "Pure gold cluster of 1:9:9:1:9:9:1 layered structure: a novel 39-metal-atom cluster $\left[\left(\mathrm{Ph}_{3} \mathrm{P}\right)_{14} \mathrm{Au}_{39} \mathrm{C}_{l 6}\right] \mathrm{C}_{l 2}$ with an interstitial gold atom in a hexagonal antiprismatic cage," Journal of the American Chemical Society, vol. 114, no. 7, pp. 2743-2745, 1992.

[94] C. E. Briant, B. R. C. Theobald, J. W. White, L. K. Bell, D. M. P. Mingos, and A. J. Welch, "Synthesis and X-ray structural characterization of the centred icosahedral gold cluster compound $\left[\mathrm{Au}_{l 3}\left(\mathrm{PMe}_{2} \mathrm{Ph}\right)_{10} \mathrm{Cl}_{2}\right]\left(\mathrm{PF}_{6}\right)_{3}$; the realization of a theoretical prediction," Journal of the Chemical Society, Chemical Communications, no. 5, pp. 201-202, 1981.

[95] G. Schmid, R. Pfeil, R. Boese et al., " $\mathrm{Au}_{55}\left[\mathrm{P}\left(\mathrm{C}_{6} \mathrm{H}_{5}\right)_{3}\right]_{12} \mathrm{Cl}_{6}$-agoldclusterofanexceptional size," Chemische Berichte/Recueil, vol. 114, no. 11, pp. 3634-3642, 1981.

[96] W. W. Weare, S. M. Reed, M. G. Warner, and J. E. Hutchison, "Improved synthesis of small (d(CORE)approximateto $1.5 \mathrm{~nm}$ ) phosphine-stabilized gold nanoparticies," Journal of the American Chemical Society, vol. 122, no. 51, pp. 12890-12891, 2000.

[97] X. K. Wan, S. F. Yuan, Z. W. Lin, and Q. M. Wang, "A chiral gold nanocluster $\mathrm{Au}_{20}$ protected by tetradentate phosphine ligands," Angewandte Chemie - International Edition, vol. 53, no. 11, pp. 2923-2926, 2014.

[98] G. H. Woehrle, L. O. Brown, and J. E. Hutchison, "Thiolfunctionalized, $1.5-\mathrm{nm}$ gold nanoparticles through ligand exchange reactions: scope and mechanism of ligand exchange," Journal of the American Chemical Society, vol. 127, no. 7, pp. 2172-2183, 2005.

[99] M. Brust, M. Walker, D. Bethell, D. J. Schiffrin, and R. Whyman, "Synthesis of thiol-derivatised gold nanoparticles in a twophase liquid-liquid system," Journal of the Chemical Society, Chemical Communications, no. 7, pp. 801-802, 1994.

[100] R. Jin, H. Qian, Z. Wu et al., "Size focusing: a methodology for synthesizing atomically precise gold nanoclusters," Journal of Physical Chemistry Letters, vol. 1, no. 19, pp. 2903-2910, 2010.

[101] Z. Wu, J. Suhan, and R. Jin, "One-pot synthesis of atomically monodisperse, thiol-functionalized $\mathrm{Au}_{25}$ nanoclusters," Journal of Materials Chemistry, vol. 19, no. 5, pp. 622-626, 2009.

[102] T. G. Schaaff, G. Knight, M. N. Shafigullin, R. F. Borkman, and R. L. Whetten, "Isolation and selected properties of a 10.4 $\mathrm{kDa}$ gold: glutathione cluster compound," Journal of Physical Chemistry B, vol. 102, no. 52, pp. 10643-10646, 1998. 
[103] Y. Negishi, Y. Takasugi, S. Sato, H. Yao, K. Kimura, and T. Tsukuda, "Magic-numbered $\mathrm{Au}_{n}$ clusters protected by glutathione monolayers $(n=18,21,25,28,32,39)$ : Isolation and spectroscopic characterization," Journal of the American Chemical Society, vol. 126, no. 21, pp. 6518-6519, 2004.

[104] Y. Negishi, K. Nobusada, and T. Tsukuda, "Glutathioneprotected gold clusters revisited: bridging the gap between gold(I)-thiolate complexes and thiolate-protected gold nanocrystals," Journal of the American Chemical Society, vol. 127, no. 14, pp. 5261-5270, 2005.

[105] H. Zhang, Q. Liu, T. Wang et al., "Facile preparation of glutathione-stabilized gold nanoclusters for selective determination of chromium (III) and chromium (VI) in environmental water samples," Analytica Chimica Acta, vol. 770, pp. 140-146, 2013.

[106] A. C. Templeton, S. Chen, S. M. Gross, and R. W. Murray, "Water-soluble, isolable gold clusters protected by tiopronin and coenzyme a monolayers," Langmuir, vol. 15, no. 1, pp. 66-76, 1999.

[107] D. Lee, R. L. Donkers, G. Wang, A. S. Harper, and R. W. Murray, "Electrochemistry and optical absorbance and luminescence of molecule-like Au38 nanoparticles," Journal of the American Chemical Society, vol. 126, no. 19, pp. 6193-6199, 2004.

[108] M. C. Paau, C. K. Lo, X. Yang, and M. M. F. Choi, "Synthesis of $1.4 \mathrm{~nm} \alpha$-cyclodextrin-protected gold nanoparticles for luminescence sensing of mercury(II) with picomolar detection limit," Journal of Physical Chemistry C, vol. 114, no. 38, pp. 1599516003, 2010.

[109] E. S. Shibu and T. Pradeep, "Quantum clusters in cavities: trapped $\mathrm{Au}_{15}$ in cyclodextrins," Chemistry of Materials, vol. 23, no. 4, pp. 989-999, 2011.

[110] H. Zhang, X. Huang, L. Li et al., "Photoreductive synthesis of water-soluble fluorescent metal nanoclusters," Chemical Communications, vol. 48, no. 4, pp. 567-569, 2012.

[111] Y. Shiraishi, D. Arakawa, and N. Toshima, "pH-dependent color change of colloidal dispersions of gold nanoclusters: effect of stabilizer," European Physical Journal E, vol. 8, no. 4, pp. 377383, 2002.

[112] L. Shang, N. Azadfar, F. Stockmar et al., "One-pot synthesis of near-infrared fluorescent gold clusters for cellular fluorescence lifetime imaging," Small, vol. 7, no. 18, pp. 2614-2620, 2011.

[113] N. K. Chaki, P. Singh, C. V. Dharmadhikari, and K. P. Vijayamohanan, "Single-electron charging features of larger, dodecanethiol-protected gold nanoclusters: electrochemical and scanning tunneling microscopy studies," Langmuir, vol. 20, no. 23, pp. 10208-10217, 2004.

[114] H. Yao, K. Miki, N. Nishida, A. Sasaki, and K. Kimura, "Large optical activity of gold nanocluster enantiomers induced by a pair of optically active penicillamines," Journal of the American Chemical Society, vol. 127, no. 44, pp. 15536-15543, 2005.

[115] X. Yang, L. Gan, L. Han, D. Li, J. Wang, and E. Wang, "Facile preparation of chiral penicillamine protected gold nanoclusters and their applications in cell imaging," Chemical Communications, vol. 49, no. 23, pp. 2302-2304, 2013.

[116] M. A. H. Muhammed, S. Ramesh, S. S. Sinha, S. K. Pal, and T. Pradeep, "Two distinct fluorescent quantum clusters of gold starting from metallic nanoparticles by $\mathrm{pH}$-dependent ligand etching," Nano Research, vol. 1, no. 4, pp. 333-340, 2008.

[117] H. Qian, Y. Zhu, and R. Jin, "Size-Focusing synthesis, optical and electrochemical properties of monodisperse $\mathrm{Au}_{38}\left(\mathrm{SC}_{2} \mathrm{H}_{4} \mathrm{Ph}\right)_{24}$ nanoclusters," ACS Nano, vol. 3, no. 11, pp. 3795-3803, 2009.
[118] H. Qian, M. Zhu, Z. Wu, and R. Jin, "Quantum sized gold nanoclusters with atomic precision," Accounts of Chemical Research, vol. 45, no. 9, pp. 1470-1479, 2012.

[119] T. G. Schaaff and R. L. Whetten, "Controlled Etching of Au: SR cluster Compounds," Journal of Physical Chemistry B, vol. 103, no. 44, pp. 9394-9396, 1999.

[120] R. A. Sperling and W. J. Parak, "Surface modification, functionalization and bioconjugation of colloidal Inorganic nanoparticles," Philosophical Transactions of the Royal Society A: Mathematical, Physical and Engineering Sciences, vol. 368, no. 1915, pp. 1333-1383, 2010.

[121] S. Jiang, K. Y. Win, S. Liu, C. P. Teng, Y. Zheng, and M.-Y. Han, "Surface-functionalized nanoparticles for biosensing and imaging-guided therapeutics," Nanoscale, vol. 5, no. 8, pp. 31273148, 2013.

[122] N. Erathodiyil and J. Y. Ying, "Functionalization of inorganic nanoparticles for bioimaging applications," Accounts of Chemical Research, vol. 44, no. 10, pp. 925-935, 2011.

[123] X. Le Guével, B. Hötzer, G. Jung, and M. Schneider, "NIRemitting fluorescent gold nanoclusters doped in silica nanoparticles," Journal of Materials Chemistry, vol. 21, no. 9, pp. 29742981, 2011.

[124] C. A. J. Lin, R. A. Sperling, J. K. Li et al., "Design of an amphiphilic polymer for nanoparticle coating and functionalization," Small, vol. 4, no. 3, pp. 334-341, 2008.

[125] S.-K. Sun, L.-X. Dong, Y. Cao, H.-R. Sun, and X.-P. Yan, "Fabrication of multifunctional $\mathrm{Gd}_{2} \mathrm{O}_{3} / \mathrm{Au}$ hybrid nanoprobe via a one-step approach for near-infrared fluorescence and magnetic resonance multimodal imaging in vivo," Analytical Chemistry, vol. 85, no. 17, pp. 8436-8441, 2013.

[126] J. M. Abad, S. F. L. Mertens, M. Pita, V. M. Fernández, and D. J. Schiffrin, "Functionalization of thioctic acid-capped gold nanoparticles for specific immobilization of histidine-tagged proteins," Journal of the American Chemical Society, vol. 127, no. 15, pp. 5689-5694, 2005.

[127] F. Wen, Y. Dong, L. Feng, S. Wang, S. Zhang, and X. Zhang, "Horseradish peroxidase functionalized fluorescent gold nanoclusters for hydrogen peroxide sensing," Analytical Chemistry, vol. 83, no. 4, pp. 1193-1196, 2011.

[128] Y. Chen, Y. Wang, C. Wang et al., "Papain-directed synthesis of luminescent gold nanoclusters and the sensitive detection of $\mathrm{Cu}^{2+}$, , Journal of Colloid and Interface Science, vol. 396, pp. 6368, 2013.

[129] C. Banerjee, J. Kuchlyan, D. Banik et al., "Interaction of gold nanoclusters with IR light emitting cyanine dyes: a systematic fluorescence quenching study," Physical Chemistry Chemical Physics, vol. 16, no. 32, pp. 17272-17283, 2014.

[130] Z. Wang, J. H. Lee, and Y. Lu, "Highly sensitive 'turn-on' fluorescent sensor for $\mathrm{Hg}^{2+}$ in aqueous solution based on structure-switching DNA," Chemical Communications, no. 45, pp. 6005-6007, 2008.

[131] Z. Gu, M. Zhao, Y. Sheng, L. A. Bentolila, and Y. Tang, "Detection of mercury ion by infrared fluorescent protein and its hydrogel-based paper assay," Analytical Chemistry, vol. 83, no. 6, pp. 2324-2329, 2011.

[132] M. Matsushita, M. M. Meijler, P. Wirsching, R. A. Lerner, and K. D. Janda, "A blue fluorescent antibody-cofactor sensor for mercury," Organic Letters, vol. 7, no. 22, pp. 4943-4946, 2005.

[133] B. Ruttkay-Nedecky, J. Kudr, L. Nejdl, D. Maskova, R. Kizek, and V. Adam, "G-quadruplexes as sensing probes," Molecules, vol. 18, no. 12, pp. 14760-14779, 2013. 
[134] J. Liu and Y. Lu, "Rational Design of 'turn-On' allosteric DNAzyme catalytic beacons for aqueous mercury ions with ultrahigh sensitivity and selectivity," Angewandte Chemie, vol. 46, no. 40, pp. 7587-7590, 2007.

[135] P. J. J. Huang and J. Liu, "Sensing parts-per-trillion $\mathrm{Cd}^{2+}, \mathrm{Hg}^{2+}$, and $\mathrm{Pb}^{2+}$ collectively and individually using phosphorothioate DNAzymes," Analytical Chemistry, vol. 86, no. 12, pp. 59996005, 2014.

[136] B.-C. Ye and B.-C. Yin, "Highly sensitive detection of mercury(II) ions by fluorescence polarization enhanced by gold nanoparticles," Angewandte Chemie International Edition, vol. 47, no. 44, pp. 8386-8389, 2008.

[137] C.-W. Tseng, H.-Y. Chang, J.-Y. Chang, and C.-C. Huang, "Detection of mercury ions based on mercury-induced switching of enzyme-like activity of platinum/gold nanoparticles," Nanoscale, vol. 4, no. 21, pp. 6823-6830, 2012.

[138] M. Suresh, S. K. Mishra, S. Mishra, and A. Das, "The detection of $\mathrm{Hg}^{2+}$ by cyanobacteria in aqueous media," Chemical Communications, no. 18, pp. 2496-2498, 2009.

[139] Nuriman, B. Kuswandi, and W. Verboom, "Selective chemosensor for $\mathrm{Hg}(\mathrm{II})$ ions based on tris[2-(4-phenyldiazenyl)phenylaminoethoxy]cyclotriveratrylene in aqueous samples," Analytica Chimica Acta, vol. 655, no. 1-2, pp. 75-79, 2009.

[140] S. Voutsadaki, G. K. Tsikalas, E. Klontzas, G. E. Froudakis, and H. E. Katerinopoulos, “A 'turn-on' coumarin-based fluorescent sensor with high selectivity for mercury ions in aqueous media," Chemical Communications, vol. 46, no. 19, pp. 3292-3294, 2010.

[141] J. Xie, Y. Zheng, and J. Y. Ying, "Highly selective and ultrasensitive detection of $\mathrm{Hg}^{2+}$ based on fluorescence quenching of $\mathrm{Au}$ nanoclusters by $\mathrm{Hg}^{2+}-\mathrm{Au}^{+}$interactions," Chemical Communications, vol. 46, no. 6, pp. 961-963, 2010.

[142] T. Mayr, I. Klimant, O. S. Wolfbeis, and T. Werner, "Dual lifetime referenced optical sensor membrane for the determination of copper(II) ions," Analytica Chimica Acta, vol. 462, no. 1, pp. 110, 2002.

[143] M. E. Letelier, A. M. Lepe, M. Faúndez et al., "Possible mechanisms underlying copper-induced damage in biological membranes leading to cellular toxicity," Chemico-Biological Interactions, vol. 151, no. 2, pp. 71-82, 2005.

[144] L. M. Gaetke and C. K. Chow, "Copper toxicity, oxidative stress, and antioxidant nutrients," Toxicology, vol. 189, no. 1-2, pp. 147$163,2003$.

[145] W. Chen, X. Tu, and X. Guo, "Fluorescent gold nanoparticlesbased fluorescence sensor for $\mathrm{Cu}^{2+}$ ions," Chemical Communications, no. 13, pp. 1736-1738, 2009.

[146] X. Tu, W. Chen, and X. Guo, "Facile one-pot synthesis of nearinfrared luminescent gold nanoparticles for sensing copper (II)," Nanotechnology, vol. 22, no. 9, Article ID 095701, 2011.

[147] Y. Xu, X. Yang, S. Zhu, and Y. Dou, "Selectively fluorescent sensing of $\mathrm{Cu}^{2+}$ based on lysine-functionalized gold nanoclusters," Colloids and Surfaces A: Physicochemical and Engineering Aspects, vol. 450, pp. 115-120, 2014.

[148] D. Cao, J. Fan, J. Qiu, Y. Tu, and J. Yan, "Masking method for improving selectivity of gold nanoclusters in fluorescence determination of mercury and copper ions," Biosensors \& Bioelectronics, vol. 42, no. 1, pp. 47-50, 2013.

[149] J.-A. Annie Ho, H.-C. Chang, and W.-T. Su, "DOPA-mediated reduction allows the facile synthesis of fluorescent gold nanoclusters for use as sensing probes for ferric ions," Analytical Chemistry, vol. 84, no. 7, pp. 3246-3253, 2012.
[150] Y.-C. Shiang, C.-C. Huang, and H.-T. Chang, "Gold nanodotbased luminescent sensor for the detection of hydrogen peroxide and glucose," Chemical Communications, no. 23, pp. 3437$3439,2009$.

[151] L. Hu, S. Han, S. Parveen, Y. Yuan, L. Zhang, and G. Xu, "Highly sensitive fluorescent detection of trypsin based on BSAstabilized gold nanoclusters," Biosensors \& Bioelectronics, vol. 32, no. 1, pp. 297-299, 2012.

[152] Q. Wen, Y. Gu, L. J. Tang, R. Q. Yu, and J.-H. Jiang, "Peptidetemplated gold nanocluster beacon as a sensitive, label-free sensor for protein post-translational modification enzymes," Analytical Chemistry, vol. 85, no. 24, pp. 11681-11685, 2013.

[153] X. Yang, Y. Luo, Y. Zhuo, Y. Feng, and S. Zhu, "Novel synthesis of gold nanoclusters templated with l-tyrosine for selective analyzing tyrosinase," Analytica Chimica Acta, vol. 840, pp. 8792, 2014.

[154] B. Aswathy and G. Sony, " $\mathrm{Cu}^{2+}$ modulated BSA-Au nanoclusters: a versatile fluorescence turn-on sensor for dopamine," Microchemical Journal, vol. 116, pp. 151-156, 2014.

[155] Y. Tao, Y. Lin, J. Ren, and X. Qu, "A dual fluorometric and colorimetric sensor for dopamine based on BSA-stabilized Au nanoclusters," Biosensors \& Bioelectronics, vol. 42, no. 1, pp. 4146, 2013.

[156] L. Li, H. Liu, Y. Shen, J. Zhang, and J. J. Zhu, "Electrogenerated chemiluminescence of $\mathrm{Au}$ nanoclusters for the detection of dopamine," Analytical Chemistry, vol. 83, no. 3, pp. 661-665, 2011.

[157] H. Liu, G. Yang, E. S. Abdel-Halim, and J.-J. Zhu, "Highly selective and ultrasensitive detection of nitrite based on fluorescent gold nanoclusters," Talanta, vol. 104, pp. 135-139, 2013.

[158] X. Chen and G. A. Baker, "Cholesterol determination using protein-templated fluorescent gold nanocluster probes," Analyst, vol. 138, no. 24, pp. 7299-7302, 2013.

[159] B. Hemmateenejad, F. Shakerizadeh-Shirazi, and F. Samari, "BSA-modified gold nanoclusters for sensing of folic acid," Sensors and Actuators, B: Chemical, vol. 199, pp. 42-46, 2014.

[160] Z. Chen, S. Qian, J. Chen, J. Cai, S. Wu, and Z. Cai, "Proteintemplated gold nanoclusters based sensor for off-on detection of ciprofloxacin with a high selectivity," Talanta, vol. 94, pp. 240245, 2012.

[161] G.-H. Yang, J.-J. Shi, S. Wang et al., "Fabrication of a boron nitride-gold nanocluster composite and its versatile application for immunoassays," Chemical Communications, vol. 49, no. 91, pp. 10757-10759, 2013.

[162] R. C. Triulzi, M. Micic, S. Giordani, M. Serry, W. A. Chiou, and R. M. Leblanc, "Immunoasssay based on the antibodyconjugated PAMAM-dendrimer-gold quantum dot complex," Chemical Communications, no. 48, pp. 5068-5070, 2006.

[163] H. Lin, L. Li, C. Lei et al., "Immune-independent and labelfree fluorescent assay for Cystatin C detection based on proteinstabilized Au nanoclusters," Biosensors and Bioelectronics, vol. 41, no. 1, pp. 256-261, 2013.

[164] K. Selvaprakash and Y.-C. Chen, "Using protein-encapsulated gold nanoclusters as photoluminescent sensing probes for biomolecules," Biosensors \& Bioelectronics, vol. 61, pp. 88-94, 2014.

[165] C.-C. Huang, C.-K. Chiang, Z.-H. Lin, K.-H. Lee, and H.T. Chang, "Bioconjugated gold nanodots and nanoparticles for protein assays based on photoluminescence quenching," Analytical Chemistry, vol. 80, no. 5, pp. 1497-1504, 2008. 
[166] C. C. Huang, C. T. Chen, Y. C. Shiang, Z. H. Lin, and H. T. Chang, "Synthesis of fluorescent carbohydrate-protected Au nanodots for detection of Concanavalin A and Escherichia coli," Analytical Chemistry, vol. 81, no. 3, pp. 875-882, 2009.

[167] J. Zhang, M. Sajid, N. Na, L. Huang, D. He, and J. Ouyang, "The application of Au nanoclusters in the fluorescence imaging of human serum proteins after native PAGE: enhancing detection by low-temperature plasma treatment," Biosensors and Bioelectronics, vol. 35, no. 1, pp. 313-318, 2012.

[168] A. M. P. Hussain, S. N. Sarangi, J. A. Kesarwani, and S. N. Sahu, "Au-nanocluster emission based glucose sensing," Biosensors \& Bioelectronics, vol. 29, no. 1, pp. 60-65, 2011.

[169] L. Jin, L. Shang, S. Guo et al., "Biomolecule-stabilized Au nanoclusters as a fluorescence probe for sensitive detection of glucose," Biosensors \& Bioelectronics, vol. 26, no. 5, pp. 19651969, 2011.

[170] W. J. Stark, "Nanoparticles in biological systems," Angewandte Chemie International Edition, vol. 50, no. 6, pp. 1242-1258, 2011.

[171] L. Shang and G. U. Nienhaus, "Small fluorescent nanoparticles at the nano-bio interface," Materials Today, vol. 16, no. 3, pp. 5866, 2013.

[172] H.-H. Wang, C. A. J. Lin, C. H. Lee et al., "Fluorescent gold nanoclusters as a biocompatible marker for in vitro and in vivo tracking of endothelial cells," ACS Nano, vol. 5, no. 6, pp. 43374344, 2011.

[173] P.-H. Chan, S.-Y. Wong, S.-H. Lin, and Y.-C. Chen, "Lysozymeencapsulated gold nanocluster-based affinity mass spectrometry for pathogenic bacteria," Rapid Communications in Mass Spectrometry, vol. 27, no. 19, pp. 2143-2148, 2013.

[174] D. Hu, Z. Sheng, S. Fang et al., "Folate receptor-targeting gold nanoclusters as fluorescence enzyme mimetic nanoprobes for tumor molecular colocalization diagnosis," Theranostics, vol. 4, no. 2, pp. 142-153, 2014.

[175] P. Botella, Í. Ortega, M. Quesada et al., "Multifunctional hybrid materials for combined photo and chemotherapy of cancer," Dalton Transactions, vol. 41, no. 31, pp. 9286-9296, 2012.

[176] P. Huang, J. Lin, S. Wang et al., "Photosensitizer-conjugated silica-coated gold nanoclusters for fluorescence imagingguided photodynamic therapy," Biomaterials, vol. 34, no. 19, pp. 4643-4654, 2013.

[177] Y. Wang, J. Chen, and J. Irudayaraj, "Nuclear targeting dynamics of gold nanoclusters for enhanced therapy of HER $2^{+}$breast cancer," ACS Nano, vol. 5, no. 12, pp. 9718-9725, 2011.

[178] X. Wang, X. Cai, J. Hu et al., 'Glutathione-triggered 'Off-On' release of anticancer drugs from dendrimer-encapsulated gold nanoparticles," Journal of the American Chemical Society, vol. 135, no. 26, pp. 9805-9810, 2013.

[179] C.-L. Liu, M.-L. Ho, Y.-C. Chen et al., "Thiol-functionalized gold nanodots: two-photon absorption property and imaging in vitro," Journal of Physical Chemistry C, vol. 113, no. 50, pp. 21082-21089, 2009.

[180] Z. Zhou, C. Zhang, Q. Qian et al., "Folic acid-conjugated silica capped gold nanoclusters for targeted fluorescence/X-ray computed tomography imaging," Journal of Nanobiotechnology, vol. 11, article 17, 2013.

[181] J. Wang, G. Zhang, Q. Li et al., "In vivo self-bio-imaging of tumors through in situ biosynthesized fluorescent gold nanoclusters," Scientific reports, vol. 3, 2013.

[182] G. Ramakrishna, O. Varnavski, J. Kim, D. Lee, and T. Goodson, "Quantum-sized gold clusters as efficient two-photon absorbers," Journal of the American Chemical Society, vol. 130, no. 15 , pp. 5032-5033, 2008.
[183] L. Polavarapu, M. Manna, and Q.-H. Xu, "Biocompatible glutathione capped gold clusters as one- and two-photon excitation fluorescence contrast agents for live cells imaging," Nanoscale, vol. 3, no. 2, pp. 429-434, 2011.

[184] T. W. J. Gadella Jr., T. M. Jovin, and R. M. Clegg, "Fluorescence lifetime imaging microscopy (FLIM): spatial resolution of microstructures on the nanosecond time scale," Biophysical Chemistry, vol. 48, no. 2, pp. 221-239, 1993.

[185] A. Zhang, Y. Tu, S. Qin et al., "Gold nanoclusters as contrast agents for fluorescent and X-ray dual-modality imaging," Journal of Colloid and Interface Science, vol. 372, no. 1, pp. 239-244, 2012.

[186] A. Retnakumari, S. Setua, D. Menon et al., "Molecular-receptorspecific, non-toxic, near-infrared-emitting Au cluster-protein nanoconjugates for targeted cancer imaging," Nanotechnology, vol. 21, no. 5, Article ID 055103, 2010. 

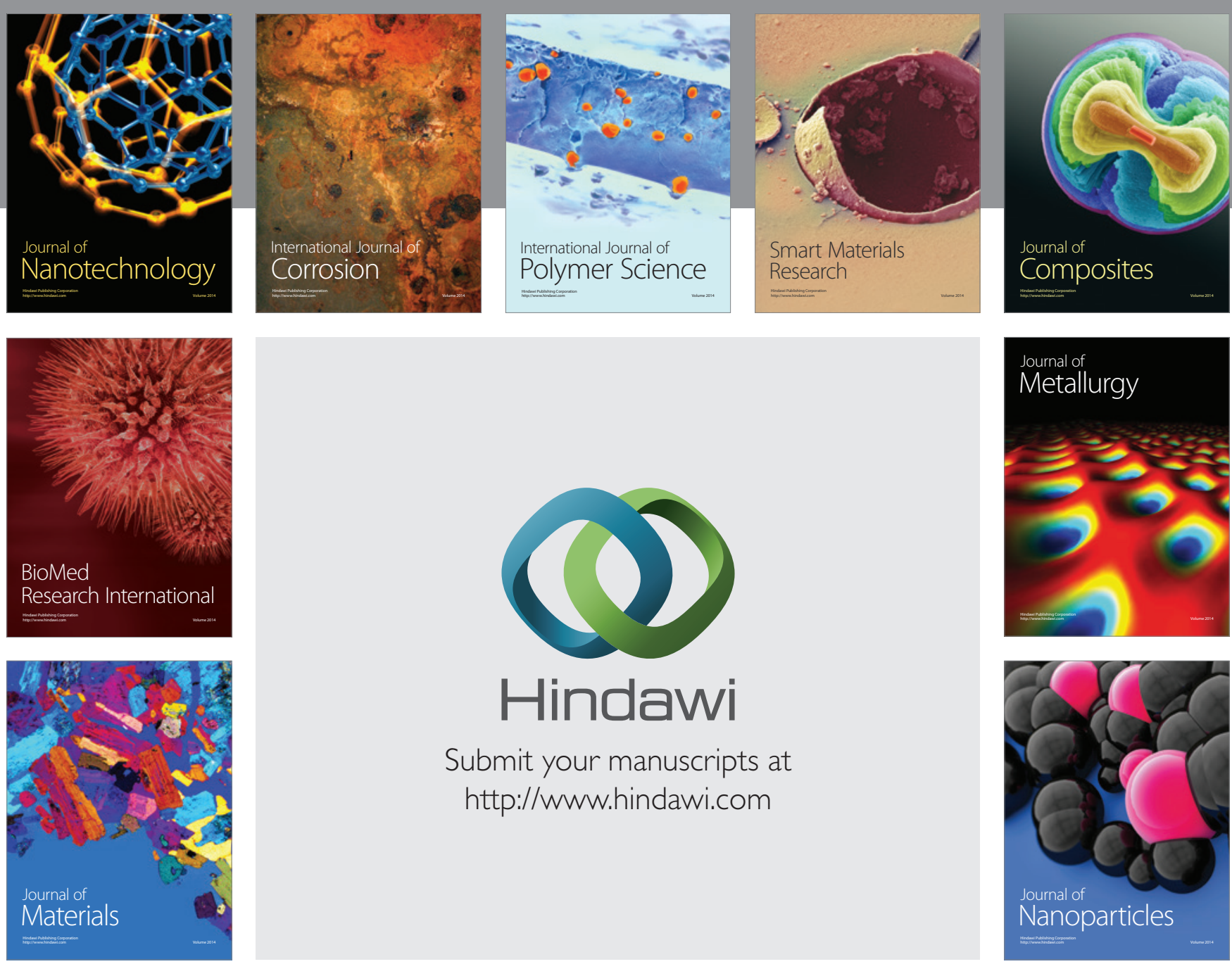

Submit your manuscripts at http://www.hindawi.com
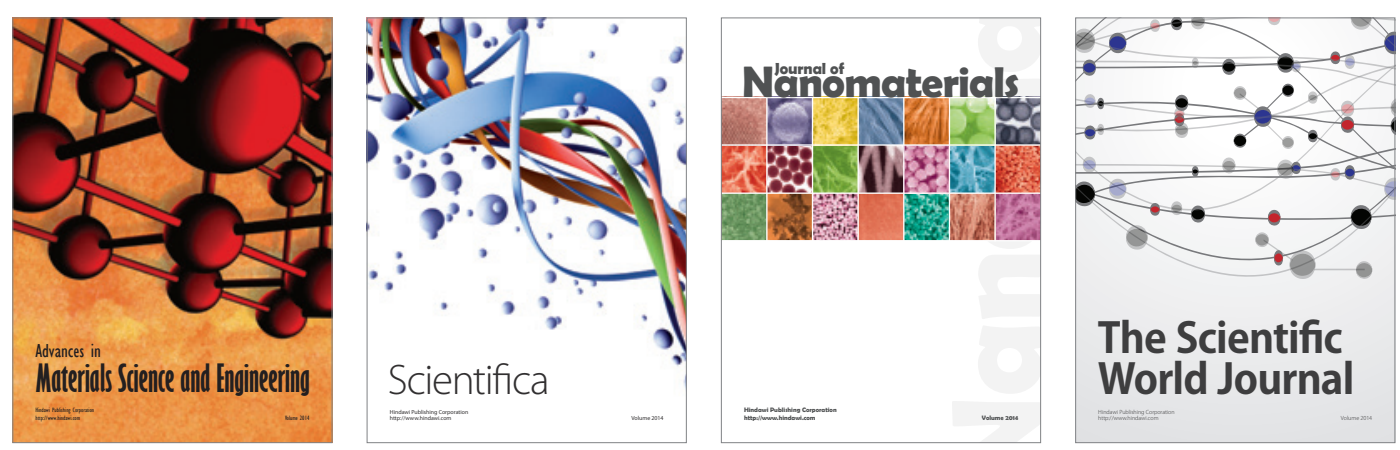

\section{The Scientific World Journal}
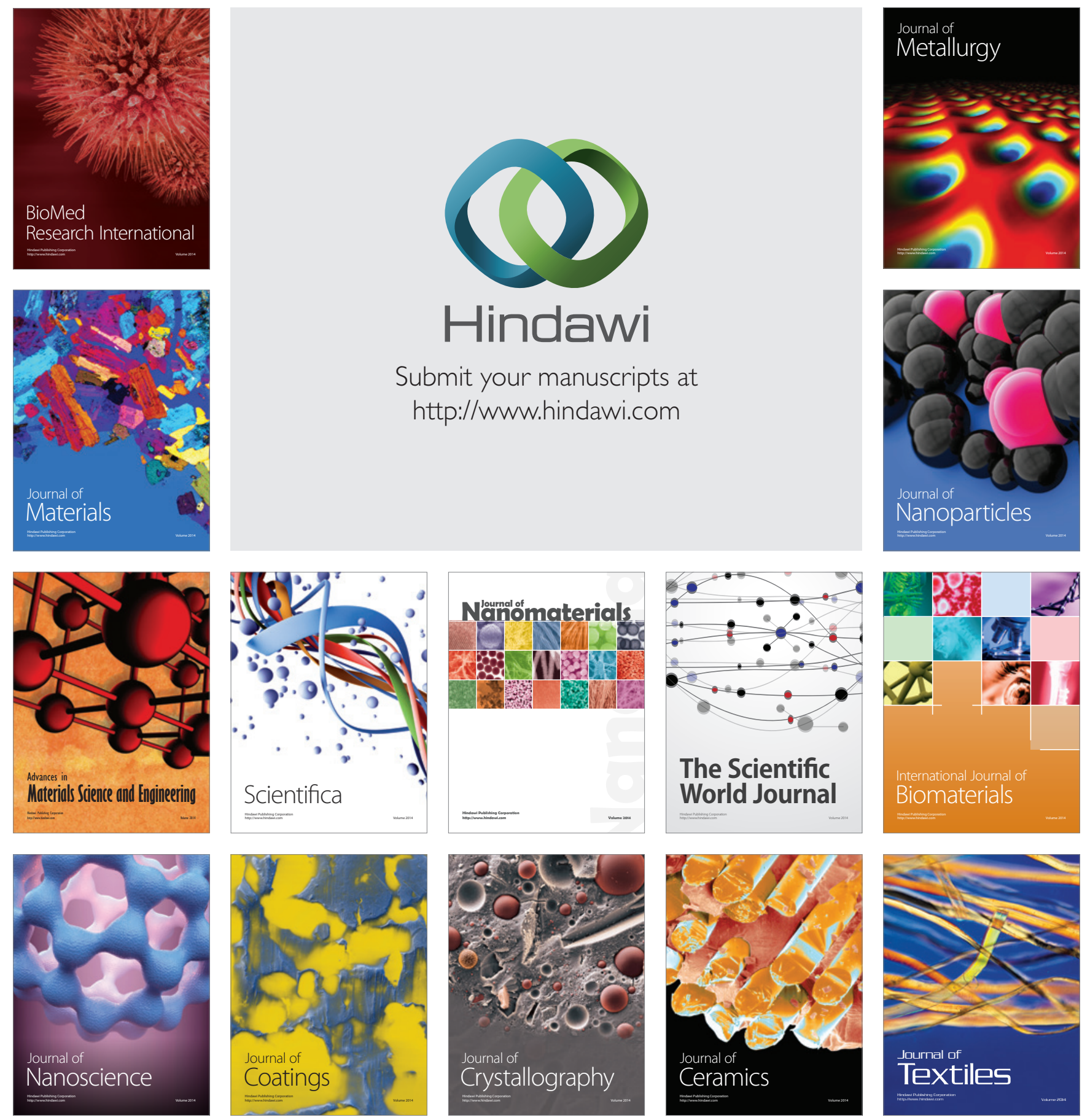